\title{
Compact stellar model in Tolman space-time in presence of pressure anisotropy
}

\author{
Piyali Bhar, ${ }^{1, *}$ Shyam Das, ${ }^{2, \dagger}$ and Bikram Keshari Parida ${ }^{3, \ddagger}$ \\ ${ }^{1}$ Department of Mathematics, Government General Degree College, Singur, Hooghly, West Bengal 712 409, India \\ ${ }^{2}$ Department of Physics, P. D. Women's College, Jalpaiguri, West Bengal 735101, India \\ ${ }^{3}$ Department of Physics, Pondicherry University, Kalapet, Puducherry 605014, India
}

\begin{abstract}
In this paper, we develop a new relativistic compact stellar model for a spherically symmetric anisotropic matter distribution. The model has been obtained through generating a new class of solutions by invoking the Tolman ansatz for one of the metric potentials $g_{r r}$ and a physically reasonable selective profile of radial pressure. We have matched our obtained interior solution to the Schwarzschild exterior spacetime over the bounding surface of the compact star. These matching conditions together with the condition of vanishing the radial pressure across the boundary of the star have been utilized to determine the model parameters. We have shown that the central pressure of the star depends on the parameter $p_{0}$. We have estimated the range of $p_{0}$ by using the recent data of compact stars $4 \mathrm{U} 1608-52$ and Vela X-1. The effect of $p_{0}$ on different physical parameters e.g., pressure anisotropy, the subliminal velocity of sound, relativistic adiabatic index etc. have also been discussed. The developed model of the compact star is elaborately discussed both analytically and graphically to justify that it satisfies all the criteria demanded a realistic star. From our analysis, we have shown that the effect of anisotropy becomes small for higher values of $p_{0}$. The mass-radius (M-R) relationship which indicates the maximum mass admissible for observed pulsars for a given surface density has also been investigated in our model. Moreover, the variation of radius and mass with central density has been shown which allow us to estimate central density for a given radius (or mass) of a compact star.
\end{abstract}

\section{INTRODUCTION}

Compact stars are the ultra-high dense objects exist in nature where a huge mass gets compacted in a small region producing the mass density beyond that of the nuclear density $[20,96,130]$. A compact star is born through a non equilibrium process of the gravitational collapse refers collectively to a neutron star, made out of neutron-rich nuclear matter or, a hybrid star made of nuclear matter with a quark matter core or, strange star exclusively composed of quark matter. White dwarfs which are less dense than neutron stars and black holes may be included in this compact star category. Compact stars are unique objects that manifest themselves across a wide range of multi-messenger signals like electromagnetic radiation from radio to gamma-rays, cosmic rays, neutrinos, and gravitational waves. It has been observed that compact stars may have huge magnetic fields, especially at the surface. Their extreme density, gravity and magnetic fields make them exceptional astrophysical laboratories for exploring the fundamental theories and interactions of elementary particles and testing general relativity at extreme conditions. Compact stars are not only extreme concerning their density but some of them also possess rotation in the millisecond regime. In fact, the first compact star has been discovered as pulsars, by observing pulsating radio signals, for the first time in 1967 [39].

The general theory of relativity (GR) attracts a lot

\footnotetext{
* piyalibhar90@gmail.com

† dasshyam321@gmail.com

‡ parida.bikram90.bkp@gmail.com
}

of interest in the fields of astrophysics, cosmology and gravitational-wave astronomy and it potentially leads to major breakthroughs since its introduction in 1915. Adoption of GR by the scientific community has increased dramatically in understanding the physics of compact stellar objects over the years. Following the discovery of quasars in the 1960s, and other very high energy phenomena in the universe such as gamma-ray bursts gravitation theory and relativistic astrophysics have gone through extensive developments in recent decades and the study of a compact star has got tremendous momentum. In relativistic astrophysics, studies of compact stars remain a field of active research since it can be used as a testbed for general relativity as well as particle behavior in the extreme conditions. Understanding the behavior and properties of stellar structure in the strong-field regime remains one of the fundamental questions.

Einstein field Equation is the cornerstone of General Relativity (GR). Exact solutions of the field equations are essential for describing the physical behavior of an astrophysical compact object. Unfortunately, the high nonlinearity associated with the field equations makes the underlying calculations complicated with mathematical difficulties. There are very few exact interior solutions of the field equations satisfying the required general physical conditions inside the star. Delgaty and Lake [15] have found 127 solutions out of which only 16 qualify the test to meets all the conditions required for describing a physically realistic system. The study of general relativistic compact stellar objects via finding exact solutions of the field equations compatible with observational data has remained one of the major research areas in relativistic astrophysics. Observational data available with the advent of gravitational wave astronomy (LIGO, Virgo, KAGRA, 
LISA), as well as to high-angular-resolution observations of black hole vicinity (EHT, VLTI/GRAVITY) stimulate many researchers to contribute immensely to the theoretical development of the physics of compact objects.

For a compact star, its mass and radius are considered as the basic properties. One can connect these with the microscopic properties of nuclear and quark matter. This link is made by the equation of state (EOS) of the matter phase(s) inside the star which relates the pressure with the energy density of dense matter. For the study of compact stars, one requires proper understanding of the equation of state (EOS) corresponding to the material compositions of the star. The equation of state used to study the compact star, in particular, for an estimation of the accurate size, maximum mass and other physical features. With the knowledge of the EOS with proper boundary conditions, physical properties (such as massradius relationships) of the star can be analyzed by integrating the equation of hydrostatic equilibrium known as Tolman-Oppenheimer-Volkoff [82] equations. In particular, a stiff equation of state produces a large maximum mass.

In literature, assuming the different form of an equation of state (EOS) researchers generated many exact solutions to the field equations for describing a realistic compact object. For example, a linear EOS have been found to develop stellar structure by many researchers $[22,63,66,98,110,115]$. Exact solutions to Einstein field equations for an anisotropic sphere admitting a quadratic type of EOS [17, 62, 106], polytropic type $[41,80,81,107,112]$ or Van der Waals type EOS [113] have also been found in the literature.

One of the main difficulty is the uncertainty estimation in the EOS, which is core to the modeling of compact stellar objects and to understand the physical behavior of a compact object. Such limitation on EOS, encourage many researchers to adopt various ad-hoc approaches for a wide variety scenario of astrophysical systems. Amongst many alternative techniques, one either assumes the geometry or the fall-off behavior of density or pressure of the matter source. Towards this direction the geometrical approach suggested by Vaidya and Tikekar [128] and Tikekar [120] having compact threespheroidal geometry or Finch-Skea ansatz [18] where the associated space-time is paraboloidal are very useful for describing a super-dense compact object. Such alternative methods are useful for the development of stellar models as shown by many investigators such as $[12,13,16,24,44,46,47,49,58,59,61,72,73,75-$ 78, 83, 85, 97, 99-101, 109, 116, 117, 119, 121-125]. Several simple but effective approaches to solve the field equations have been also used where the two metric potentials $g_{t t}$ and $g_{r r}$ are in general linked through an equation. The dependency of the metric potentials describes as Karmakar embedding class one method, conformally flat geometry and conformal motion respectively.

The first interior solution [95] corresponding to a spherically symmetric stellar object was given by Karl
Schwarzschild by imposing the isotropic condition on the Einstein equations. The matter under consideration was treated to be perfect fluid, which has an equal radial $\left(p_{r}\right)$ and tangential $\left(p_{t}\right)$ pressures. Later, Lemaitre in 1933 [54] developed a constant density anisotropic stellar model supported with tangential pressure only. This model was generalized for variable density by Florides [19]. In 1972, Ruderman [93] and Canuto [9] pointed out that at very high-density nuclear matter may become anisotropic in general. From the theoretical point of view, it is important to include the pressure anisotropy in the energy-momentum tensor describing the matter distribution of the system for describing the relativistic stellar structure. Bowers and Liang [8] generalized the equations of hydrostatic equilibrium to include the effects of local anisotropy on relativistic fluid spheres. Their work suggests that the anisotropy effects on the maximum equilibrium mass and surface redshift of a compact star. Interestingly, it was shown by Ivanov [45] that by considering a compact system to be anisotropic the effects of shear, electromagnetic field, etc. can be automatically taken into account. The different factors that have been identified for the justification of the existence of anisotropy within a stellar interior such as the presence of a solid core [48], phase transitions [103], a type III superfluid, a pion condensation [94] or the presence of the electrical field [127], slow rotation [51]. Strong magnetic fields can also generate an anisotropic pressure inside a self-gravitating body as pointed out by Weber [129]. It has been shown that the mixture of two gases can be represented by effective anisotropic fluid models [1, 55]. On the galactic scale, the existence of the anisotropy has been pointed out [5]. Several anisotropic models have been investigated by Maurya and Gupta [69], Maurya et al. [71], Pandya et al. [84], Bhar et al. [3], Murad [74], Maharaj and Mafa Takisa [60], Mafa Takisa et al. $[56,57]$, Sunzu et al. [104, 105]. General algorithms for generating static anisotropic solutions was also found by Lake[53]. Some recent work may be found in Refs. $[28,29,50,64,65,67,68,70,108,111,114]$. A complete review on anisotropic fluid spheres can be found by the work of Herrera and Santos [38].

As far as the dynamical evolution of a self-gravitating system is concerned the anisotropic effects on the evolution was investigated by Herrera and co-workers [31-33], Chan et al. [10], Govinder et al. [23], Herrera and Santos [34], Chan et al. [11], Naidu et al. [79] and Rajah and Maharaj [87]. Conformally flat solutions corresponding to anisotropic compact self-gravitating objects were developed by Herrera et al. [36, 37]. Raposo et al. also [88] studied on the dynamical properties of anisotropic self-gravitating fluids in a covariant framework.

Inspired by the previous work done by several researchers, in the present paper we develop a model of a compact star by assuming a physically reasonable choice for the radial pressure. Our paper has been organized as follows. In sect. II Einstein field equations have been described. Sect. III deals with the solutions of the field 
equation. In the next section (sect. IV) we have matched our interior solution to the exterior Schwarzschild spacetime at the boundary. The physical attributes and the stability condition of the model are respectively given in sect. V and VI. Some discussions and concluding remarks have been given in sect. VIII.

\section{INTERIOR SPACETIME AND EINSTEIN FIELD EQUATIONS}

It is well known that the energy-momentum tensor for an anisotropic model of the compact object can be described by,

$$
T_{\chi}^{\xi}=\left(\rho+p_{t}\right) u^{\xi} u_{\chi}+p_{t} g_{\chi}^{\xi}+\left(p_{r}-p_{t}\right) \eta^{\xi} \eta_{\chi},
$$

where $\eta^{i}$ is the space-like vector and the vector $u_{i}$ represent fluid 4-velocity and which is orthogonal to $\eta^{i}$ with $-u^{i} u_{j}=\eta^{i} \eta_{j}=1$ and $u^{i} \eta_{j}=0 . \quad \rho, p_{r}$ and $p_{t}$ are the matter density, radial pressure and tangential pressure respectively.

In curvature co-ordinate $(t, r, \theta, \phi)$ for a static spherically symmetry configuration in $(3+1)$-dimension, the interior spacetime is described by the line element,

$$
d s_{-}^{2}=-e^{\nu(r)} d t^{2}+e^{\lambda(r)} d r^{2}+r^{2}\left(d \theta^{2}+\sin ^{2} \theta d \phi^{2}\right),
$$

where $\lambda$ and $\nu$ being metric potentials and are the functions of the radial coordinate ' $\mathrm{r}$ '.

Using the Einstein field equations $G_{\mu \nu}=\kappa T_{\mu \nu}$, we get the following set of equations

$$
\begin{aligned}
\kappa c^{2} \rho & =\frac{\lambda^{\prime}}{r} e^{-\lambda}+\frac{1}{r^{2}}\left(1-e^{-\lambda}\right), \\
\kappa p_{r} & =\frac{1}{r^{2}}\left(e^{-\lambda}-1\right)+\frac{\nu^{\prime}}{r} e^{-\lambda}, \\
\kappa p_{t} & =\frac{1}{4} e^{-\lambda}\left[2 \nu^{\prime \prime}+\nu^{\prime 2}-\lambda^{\prime} \nu^{\prime}+\frac{2}{r}\left(\nu^{\prime}-\lambda^{\prime}\right)\right],
\end{aligned}
$$

where $\kappa=\frac{8 \pi G}{c^{4}}, G_{\mu \nu}$ being Einstein tensor, $G$ is the universal gravitational constant and $c$ is the speed of the light. In the above equations 'prime' denotes differentiation with respect to radial co-ordinate $r$. In this respect we want to mention that the mass function $m(r)$ inside the radius ' $\mathrm{r}$ ' can be obtained from the relation $e^{-\lambda}=1-\frac{2 m(r)}{r}$ which can directly follow from eq. (3).

\section{CHOICE OF METRIC POTENTIAL AND NEW ANISOTROPIC SOLUTION}

It is well known that the solutions of the field equation which could describe a compact stellar structure that satisfies all the physically reasonable conditions are a very difficult job. To reduce the difficulties we apply a geometrical approach by prescribing a suitable form of the metric function. To solve the above set of Eqs. (3)-(5), let us take the metric potential $g_{r r}$ in the following form:

$$
e^{\lambda}=1+a r^{2}+b r^{4}
$$
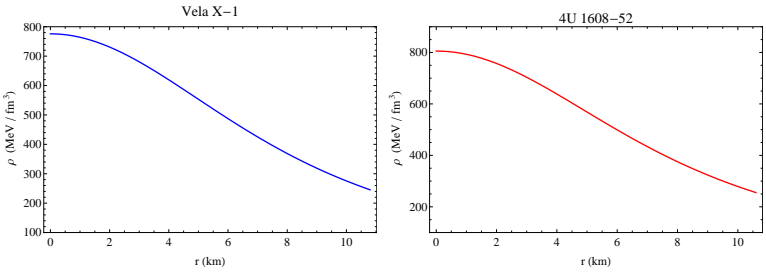

FIG. 1: Energy density $\rho$ is plotted against $r$ inside the stellar interior for a possible modelling of the compact stars Vela X-1 and 4U 1608-52.
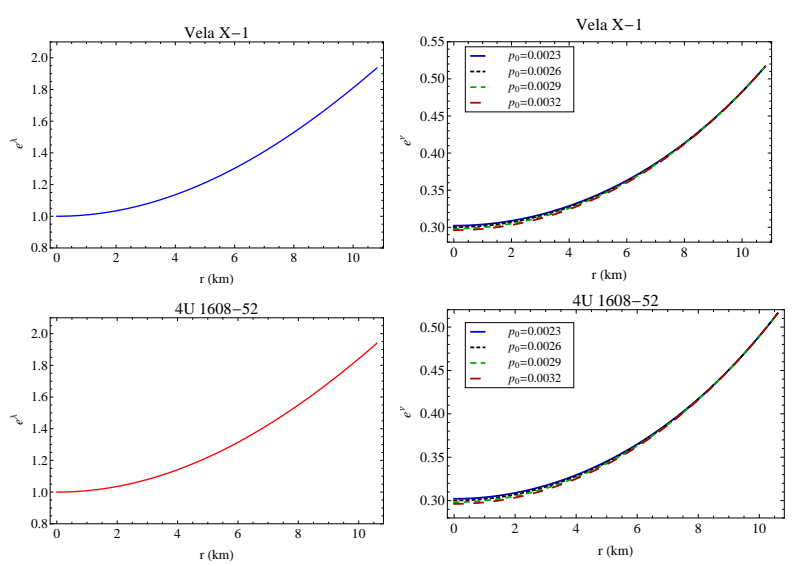

FIG. 2: The metric potentials $e^{\nu}$ and $e^{\lambda}$ are plotted against $r$ inside the stellar interior for a possible modelling of the compact stars Vela X-1 and $4 \mathrm{U}$

1608-52.

where ' $a$ ' and ' $b$ ' are constant parameters having the units $\mathrm{km}^{-2}$ and $\mathrm{km}^{-4}$ respectively. This metric potential was proposed by Tolman[126] for describing a compact stellar object. This metric potential is free from central singularity and monotonic increasing function of $r$. This type of ansatz were used earlier by several authors to model compact star in both GTR and modified gravity [4, 7].

Substituting the expression for the metric potential $e^{\lambda}$ from (6) into (3) and assuming $G=1=c$, the matter density can be obtained as,

$$
8 \pi \rho=\frac{3 a+\left(a^{2}+5 b\right) r^{2}+2 a b r^{4}+b^{2} r^{6}}{\left(1+a r^{2}+b r^{4}\right)^{2}},
$$

To find the other metric potential $e^{\nu}$ let us assume the radial pressure in the form

$$
8 \pi p_{r}=\frac{p_{0}\left(1-a r^{2}\right)}{\left(1+a r^{2}+b r^{4}\right)^{2}},
$$

where $p_{0}$ is a non-negative constant. The expression of $p_{r}$ is reasonable due to the fact that it is monotonic decreasing function of ' $r$ ' and vanishes at $r=\frac{1}{\sqrt{a}}$. Therefore, the radius of the star is obtained as $R=\frac{1}{\sqrt{a}}$, which is a finite quantity. Moreover, it possess a finite value of central pressure equal to $p_{0} / 8 \pi$ for all $p_{0}>0$. The same type of choice of pressure was earlier used by Sharma and 

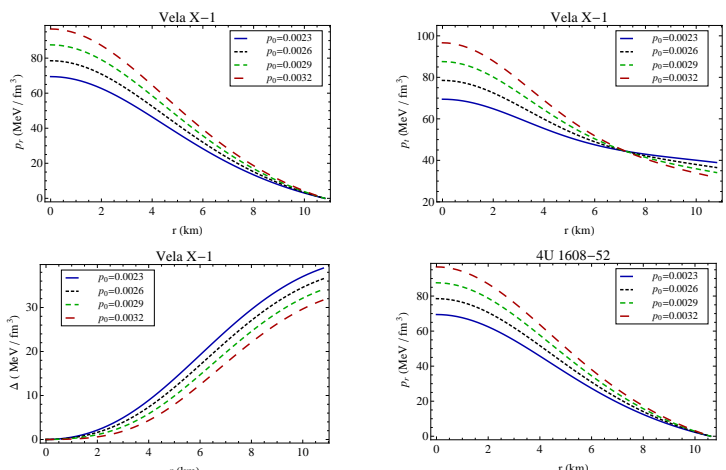

$\mathrm{r}(\mathrm{km})$
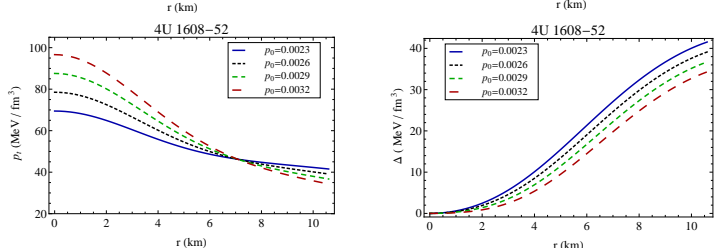

FIG. 3: Radial pressure $p_{r}$, transverse pressure $p_{t}$ and anisotropic factor $\Delta$ are plotted against $r$ inside the stellar interior for a possible modelling of the compact stars Vela X-1 and 4U 1608-52 for $p_{0}=0.0023$, $p_{0}=0.0026, p_{0}=0.0029$ and $p_{0}=0.0032$ by considering different values of 'a' and 'b' mentioned in Table II.

Ratanpal [102] for describing a static spherically symmetric anisotropic stellar configuration.

Substituting the expression for the radial pressure $p_{r}$ mentioned above and the metric potential $e^{\lambda}$ into (4) we get,

$$
\frac{d \nu}{d r}=\frac{p_{0} r\left(1-a r^{2}\right)}{1+a r^{2}+b r^{4}}+r\left(a+b r^{2}\right),
$$

On integrating equation (9) we get,

$$
\begin{aligned}
\nu= & \frac{1}{4}\left[2 a r^{2}+b r^{4}-\frac{2 p_{0}\left(a^{2}+2 b\right)}{b \sqrt{a^{2}-4 b}} \tan ^{-1}\left(\frac{a+2 b r^{2}}{\sqrt{a^{2}-4 b}}\right)\right. \\
& \left.-\frac{a p_{0}}{b} \log \left(1+a r^{2}+b r^{4}\right)\right]+B,
\end{aligned}
$$

where $B$ is the constant of integration, which will be determined from the boundary conditions. Employing the expression of the metric potentials in Eq. (5) we get the expression of the transverse pressure $p_{t}$ as,

$$
\begin{aligned}
8 \pi p_{t}= & \frac{1}{4 \Psi^{3}}\left[p_{0}^{2} r^{2}\left(1-a r^{2}\right)^{2}+r^{2}\left(a+b r^{2}\right) \Psi \xi_{1}\right. \\
& \left.-2 p_{0}\left(-2+4 a r^{2}++\xi_{2}\right)\right],
\end{aligned}
$$

where $\xi_{1}, \xi_{2}$ defined as,

$$
\begin{array}{cc}
\xi_{1} & =3 a+\left(a^{2}+5 b\right) r^{2}+2 a b r^{4}+b^{2} r^{6}, \\
\xi_{2}=\left(a^{2}+3 b\right) r^{4}+a\left(a^{2}-3 b\right) r^{6}+\left(2 a^{2}-b\right) b r^{8}+a b^{2} r^{10}, \\
\Psi & =1+a r^{2}+b r^{4} .
\end{array}
$$

The anisotropic factor $\Delta=p_{t}-p_{r}$ is given by,

$$
\begin{aligned}
8 \pi \Delta= & \frac{r^{2}}{4 \Psi^{3}}\left[A_{1}+A_{2} r^{2}+A_{3} r^{4}+A_{4} r^{6}+A_{5} r^{8}\right. \\
& \left.+4 a b^{3} r^{10}+b^{4} r^{12}\right],
\end{aligned}
$$

where $A_{i}$ 's are constants given by,

$$
\begin{aligned}
& A_{1}=3 a^{2}-8 a p_{0}+p_{0}^{2}, \\
& A_{2}=2\left(2 a^{3}+4 a b+a^{2} p_{0}-5 b p_{0}-a p_{0}^{2}\right), \\
& A_{3}=a^{4}+5 b^{2}-2 a^{3} p_{0}+10 a b p_{0}+a^{2}\left(14 b+p_{0}^{2}\right), \\
& A_{4}=2 b\left(2 a^{3}+8 a b-2 a^{2} p_{0}+b p_{0}\right), \\
& A_{5}=2 b^{2}\left(3\left(a^{2}+b\right)-a p_{0}\right) .
\end{aligned}
$$

The anisotropy factor $\Delta$ measures the anisotropy of the system and $\frac{2 \Delta}{r}$ is termed as the anisotropic force which will be repulsive in nature if $p_{t}>p_{r}$ and attractive if the inequalities is in reverse direction. In the coming sections we shall check the physical features of our proposed model.

\section{EXTERIOR SPACETIME AND MATCHING CONDITION}

To fix the model parameters $a, b$ and $D$ we match our interior spacetime to the exterior Schwarzschild spacetime at the boundary $r=R$, where $R$ is the radius of the star. For our present case the interior and exterior line element is given by,

$$
\begin{aligned}
d s_{-}^{2}= & -\exp \left\{\frac { 1 } { 4 } \left\{2 a r^{2}+b r^{4}+\frac{2 p_{0}\left(a^{2}+2 b\right)}{b \sqrt{a^{2}-4 b}} \times\right.\right. \\
& \left.\tan ^{-1}\left(\frac{a+2 b r^{2}}{\sqrt{a^{2}-4 b}}\right)-\frac{a p_{0}}{b} \log \left(1+a r^{2}+b r^{4}\right)\right\} \\
& +B\} d t^{2}+\left(1+a r^{2}+b r^{4}\right) d r^{2} \\
& +r^{2}\left(d \theta^{2}+\sin ^{2} \theta d \phi^{2}\right) \\
d s_{+}^{2}= & -\left(1-\frac{2 M}{r}\right) d t^{2}+\left(1-\frac{2 M}{r}\right)^{-1} d r^{2} \\
& +r^{2}\left(d \theta^{2}+\sin ^{2} \theta d \phi^{2}\right) .
\end{aligned}
$$

Now using the matching condition at the boundary of the star, one can get two fundamental form. The first fundamental from consists in the continuity of the metric potential across the boundary $r=R$. Explicitly

$$
\left.e^{\lambda^{-}}\right|_{r=R}=\left.e^{\lambda^{+}}\right|_{r=R} \quad \text { and }\left.\quad e^{\nu^{-}}\right|_{r=R}=\left.e^{\nu^{+}}\right|_{r=R} .
$$

The second fundamental form gives,

$$
p_{r}(R)=0
$$

which determines the size of the compact object. The second fundamental form tells us that the size of a compact star can not be arbitrarily large, i.e., it is finite. 
Therefore, from first fundamental form we obtain

$$
\begin{aligned}
\ln \left(1-\frac{2 M}{R}\right)= & \frac{1}{4}\left\{2 a R^{2}+b R^{4}+\frac{2 p_{0}\left(a^{2}+2 b\right)}{b \sqrt{a^{2}-4 b}} \times\right. \\
& \tan ^{-1}\left(\frac{a+2 b R^{2}}{\sqrt{a^{2}-4 b}}\right) \\
& \left.-\frac{a p_{0}}{b} \log \left(1+a R^{2}+b R^{4}\right)\right\}+B \\
\left(1-\frac{2 M}{R}\right)^{-1}= & 1+a R^{2}+b R^{4} .
\end{aligned}
$$

Using the expression of radial pressure (8) from the Eq. (16) we have,

$$
R^{2}=\frac{1}{a} .
$$

Now solving the Eqs. (17)-(19) we get,

$$
\begin{aligned}
a= & \frac{1}{R^{2}}, \\
b= & \frac{1}{R^{4}}\left[\left(1-\frac{2 M}{R}\right)^{-1}-2\right], \\
B= & \ln \left(1-\frac{2 M}{R}\right)-\frac{1}{4}\left\{2 a R^{2}+b R^{4}+\frac{2 p_{0}\left(a^{2}+2 b\right)}{b \sqrt{a^{2}-4 b}} \times\right. \\
& \left.\tan ^{-1}\left(\frac{a+2 b R^{2}}{\sqrt{a^{2}-4 b}}\right)-\frac{a p_{0}}{b} \log \left(1+a R^{2}+b R^{4}\right)\right\} .
\end{aligned}
$$

The above set of equations determines the constants $a$, $b$ and $B$ in terms of mass, radius of the compact object. So we can calculate the model parameters $a, b$ and $B$ numerically for a particular compact star from its estimated mass and radius data. For our present work we have estimated the mass and radius for the two compact stars Vela X-1[89] and 4U 1608-52 [26] which has been presented in Table I and the values of the constants $a, b$ are obtained in Table II where as the numerical values of $B$ is obtained in Table III. One interesting thing we can note that here $a, b$ do not depends on $p_{0}$ but $B$ does.

\section{ANALYSIS OF THE SOLUTION}

\section{A. Nature of pressure and density}

The density and pressure at the core of the star is obtained as,

$$
\rho_{c}=\frac{3 a}{\kappa} ; \quad p_{c}=\frac{p_{0}}{\kappa}
$$

. Now it is well known that for a physically acceptable model, both the central density and central pressure are non-negative, which gives the following two inequalities:

$$
a>0 ; \quad p_{0}>0 .
$$

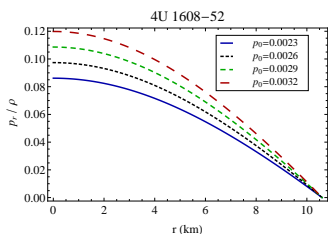

$\mathrm{r}(\mathrm{km})$
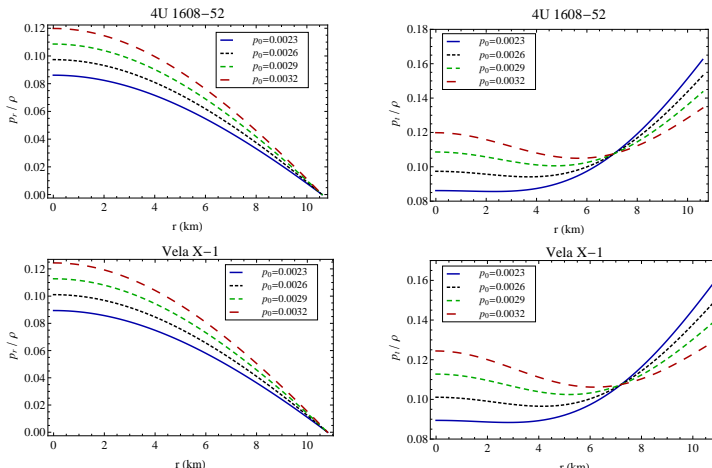

(1m)

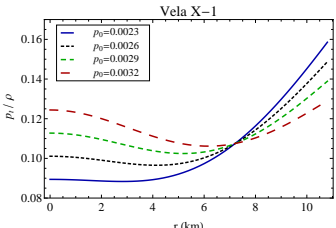

FIG. 4: The equation of state parameters are plotted against radial parameter $r$ for a possible modelling of the compact star Vela X-1 and 4U 1608-52 for different values of $p_{0}$ mentioned in the figures.

It is an important task to find a physically reasonable bound for the central density $p_{0}$ which we shall discuss in the coming sections.

The density and pressure gradients are obtained as,

$$
\begin{aligned}
\kappa \frac{d \rho}{d r}= & -\frac{2 r}{\Psi^{3}}\left(5 a^{2}-5 b+B_{1} r^{2}+B_{2} r^{4}+3 a b^{2} r^{6}+b^{3} r^{8}\right), \\
\kappa \frac{d p_{r}}{d r}= & -\frac{2 p_{0}}{\Psi^{2}}\left(2 b r^{3}+a r\left(2-b r^{4}\right)\right), \\
\kappa \frac{d p_{t}}{d r}= & \frac{r}{2 \Psi^{4}} \times\left[B_{3}+2 B_{4} r^{2}-B_{5} r^{4}-4 B_{6} r^{6}+B_{7} r^{8}\right. \\
& \left.+4 b B_{8} r^{10}+B_{9} r^{12}+B_{10} r^{14}+B_{11} r^{16}\right],
\end{aligned}
$$

where $B_{i}^{\prime} s$ are constants given by,

$$
\begin{aligned}
B_{1}= & a\left(a^{2}+13 b\right), \\
B_{2}= & 3 b\left(a^{2}+4 b\right) \\
B_{3}= & 3 a^{2}-16 a p_{0}+p_{0}^{2}, \\
B_{4}= & a^{3}+8 a b+3 a^{2} p_{0}-14 b p_{0}-2 a p_{0}^{2}, \\
B_{5}= & a^{4}-19 a^{2} b-15 b^{2}+a\left(17 a^{2}-58 b\right) p_{0} \\
& +2\left(a^{2}+b\right) p_{0}^{2}, \\
B_{6}= & -8 a b^{2}+\left(a^{4}+10 a^{2} b-9 b^{2}\right) p_{0} \\
& +a\left(-a^{2}+b\right) p_{0}^{2}, \\
B_{7}= & b\left(a^{4}+4 a^{2} b+15 b^{2}\right)-a\left(a^{4}+8 a^{2} b+49 b^{2}\right) p_{0} \\
& +\left(a^{4}+6 a^{2} b-3 b^{2}\right) p_{0}^{2}, \\
B_{8}= & a b\left(a^{2}+b\right)-\left(a^{4}+a^{2} b+2 b^{2}\right) p_{0}+a b p_{0}^{2}, \\
B_{9}= & b^{2}\left(b-a p_{0}\right)\left(b+a\left(6 a+p_{0}\right)\right), \\
B_{10}= & 4 a b^{3}\left(b-a p_{0}\right), \\
B_{11}= & b^{4}\left(b-a p_{0}\right) .
\end{aligned}
$$

Now at the center of the star,

$$
\begin{aligned}
\kappa \rho^{\prime \prime} & =-10\left(a^{2}-b\right)<0, \\
\kappa p_{r}^{\prime \prime} & =-4 a p_{0}<0, \\
\kappa p_{t}^{\prime \prime} & =\frac{3 a^{2}-16 a p_{0}+p_{0}^{2}}{2}<0 .
\end{aligned}
$$


From Eq. (24), we have,

$$
b<a^{2}
$$

and Eq. (25) is automatically satisfied. Eq. (26) implies,

$$
p_{0} \in(0.189 a, 15.81 a)
$$

The equation of state parameters $\omega_{r}$ and $\omega_{t}$ are given by,

$$
\begin{aligned}
\omega_{r} & =\frac{p_{r}}{\rho}=\frac{p_{0}\left(1-a r^{2}\right) \Psi}{\xi_{1}}, \\
\omega_{t} & =\frac{p_{t}}{\rho}=\frac{1}{4 \Psi \xi_{1}}\left[p_{0}^{2} r^{2}\left(1-a r^{2}\right)^{2} \Psi++\varphi\right],
\end{aligned}
$$

Where,

$$
\begin{aligned}
\varphi= & p_{0}\left(4-a^{4} r^{8}-3 a^{3} \xi_{3}-a^{2} \xi_{4}-a r^{2} \xi_{5}+b r^{4} \xi_{6}\right) \\
& +r^{2}\left(a+b r^{2}\right) \Psi \xi_{1}, \\
\xi_{3}= & r^{6}\left(2+b r^{4}\right), \\
\xi_{4}= & r^{4}+11 b r^{8}+3 b^{2} r^{12}, \\
\xi_{5}= & 4+b r^{4}\left(-2+b r^{4}\right)\left(6+b r^{4}\right), \\
\xi_{6}= & -2+b r^{4}\left(7+b r^{4}\right) .
\end{aligned}
$$

\section{B. Mass function and redshift}

Introducing the relation between the mass function $m(r)$ and the metric potential $e^{\lambda}, e^{-\lambda}=1-\frac{2 m}{r}$, the expression for mass function can be obtained as,

$$
m(r)=\frac{r^{3}\left(a+b r^{2}\right)}{2\left(1+a r^{2}+b r^{4}\right)} .
$$

Using the formulae of the compactness factor $u(r)$ and surface redshift $\left(z_{s}\right)$

$$
\begin{aligned}
u(r) & =\frac{m}{r}, \\
1+z_{s}(R) & =\frac{1}{\sqrt{1-2 u(R)}},
\end{aligned}
$$

the expression of these two quantities in our present model are obtained as,

$$
\begin{aligned}
u(r) & =\frac{r^{2}\left(a+b r^{2}\right)}{2\left(1+a r^{2}+b r^{4}\right)}, \\
z_{s} & =\sqrt{1+a R^{2}+b R^{4}}-1,
\end{aligned}
$$

where ' $R$ ' being the radius of the star. The gravitational redshift is obtained as,

$$
\begin{aligned}
Z= & \frac{1}{\sqrt{e^{\nu}}}-1 \\
= & {\left[\operatorname { e x p } \left\{2 a r^{2}+\frac{1}{4}\left[\frac{2 p_{0}\left(a^{2}+2 b\right)}{b \sqrt{a^{2}-4 b}} \tan ^{-1}\left(\frac{a+2 b r^{2}}{\sqrt{a^{2}-4 b}}\right)\right.\right.\right.} \\
& \left.\left.\left.+b r^{4}-\frac{a p_{0}}{b} \log \left(1+a r^{2}+b r^{4}\right)\right]+B\right\}\right]^{-\frac{1}{2}}-1
\end{aligned}
$$

Now the central value of the gravitational redshift is obtained as,

$$
Z_{0}=\frac{1}{\sqrt{\left[\exp \left\{\frac{1}{4}\left[\frac{2 p_{0}\left(a^{2}+2 b\right)}{b \sqrt{a^{2}-4 b}} \tan ^{-1}\left(\frac{a}{\sqrt{a^{2}-4 b}}\right)\right]+B\right\}\right]}}-1 .
$$

The gradient of the gravitational redshift is obtained as,

$$
\frac{d Z}{d r}=-\frac{1}{2 \sqrt{e^{\nu}}}\left[r \frac{p_{0}\left(1-a r^{2}\right)}{1+a r^{2}+b r^{4}}+r\left(a+b r^{2}\right)\right] .
$$

Now at the center of the star $\frac{d Z}{d r}=0$, and

$$
\left(\frac{d^{2} Z}{d r^{2}}\right)_{r=0}=-\frac{\left(a+p_{0}\right)}{2 \sqrt{e^{B+\frac{\left(a^{2}+2 b\right) p_{0} \tan ^{-1}\left(\frac{a}{\sqrt{a^{2}-4 b}}\right)}{2 b \sqrt{a^{2}-4 b}}}}}<0 .
$$

It verifies that the gravitational redshift has maximum value at the center of the star.

\section{Mass-radius relationship}

We have generated the mass-radius $(M-R)$ relationship for our developed model as shown in Fig. 5. The mass-radius relationship obtained for an assumed surface density $8.5 \times 10^{14} \mathrm{gm} / \mathrm{cc}$. The chosen surface density is roughly close to that considered by Sharma and Maharaj [98]. The maximum mass allowed in this model is found to be $2.7 M_{\odot}$ with the corresponding radius of value 8.9 $\mathrm{km}$. This limit on maximum mass for a neutron star is approximately $3.2 M_{\odot}[91]$.

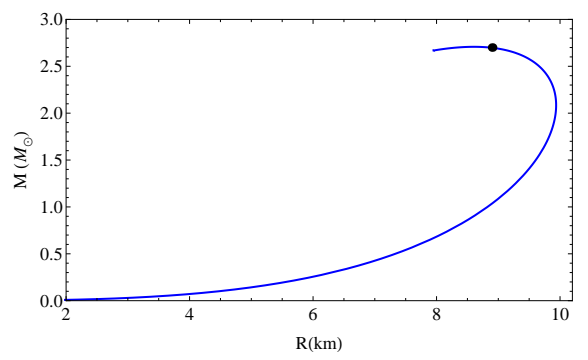

FIG. 5: Mass-radius relationship.

\section{Radius-Central density and mass-central density relation}

For compact objects we have plotted the variation of the radius and mass with the central density in Fig. 6. This plot allows us to determine the central density of compact star once the radius or the mass of the corresponding star is known. Similar type of study can be found in the work of Deb et al. [14]. 
TABLE I: The observed and estimated mass and radius for a possible modelling of the compact star 4U 1608-52 [26] and Vela X-1 [89].

\begin{tabular}{lccrc}
\hline Compact Star & $\begin{array}{c}M / M_{\odot} \\
\text { (observed) }\end{array}$ & $\begin{array}{c}\mathrm{R}(\mathrm{km}) \\
\text { (observed) }\end{array}$ & $\begin{array}{c}M / M_{\odot} \\
\text { (estimated) }\end{array}$ & $\begin{array}{c}\mathrm{R}(\mathrm{km}) \\
\text { (estimated) }\end{array}$ \\
\hline 4U 1608-52 & $1.74 \pm 0.14$ & $10.811 \pm 0.197$ & 1.74 & 10.6 \\
Vela X-1 & $1.77 \pm 0.08$ & $10.852 \pm 0.108$ & 1.77 & 10.8 \\
\hline
\end{tabular}

TABLE II: The numerical values of $a, b$ central density $\left(\rho_{c}\right)$ and surface density $\left(\rho_{s}\right)$ are obtained for a possible modelling of the compact star $4 \mathrm{U} 1608-52$ [26] and Vela X-1 [89].

\begin{tabular}{lrrrr}
\hline $\begin{array}{l}\text { Compact Star } \\
\text { units }\end{array}$ & $a$ & $b$ & $\rho_{c}$ & $\rho_{s}$ \\
$\mathrm{~km}^{-2}$ & $\mathrm{~km}^{-4}$ & ${\mathrm{gm} . \mathrm{cm}^{-3}}^{15}$ & $\mathrm{gm}^{-3}$ \\
\hline 4U 1608-52 & 0.00889996 & $-4.8392 \times 10^{-6}$ & $1.43346 \times 10^{15}$ & $4.54525 \times 10^{14}$ \\
Vela X-1 & 0.00857339 & $-4.70387 \times 10^{-6}$ & $1.38086 \times 10^{15}$ & $4.36711 \times 10^{14}$ \\
\hline
\end{tabular}
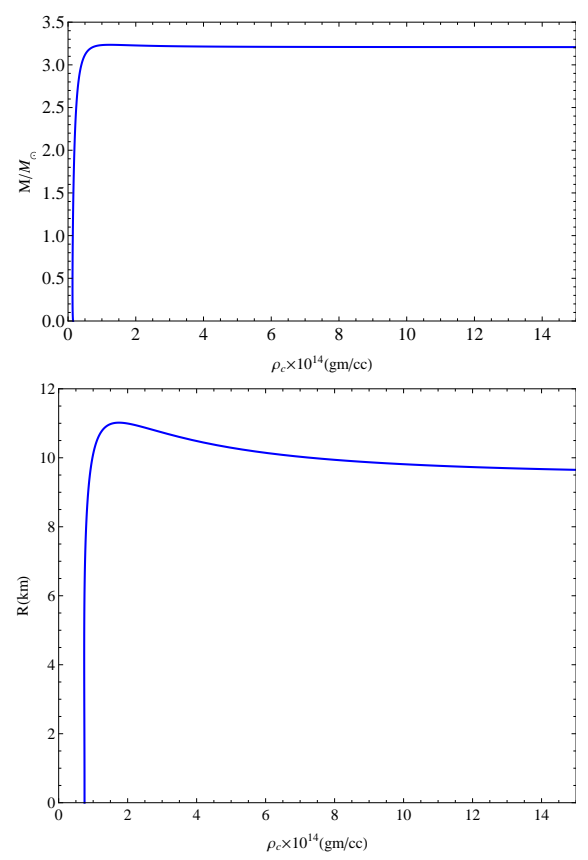

FIG. 6: Variation of radius and mass with central density.

\section{E. Energy Conditions}

It is well known that for a compact star model, the energy conditions should be satisfied and in this subsection we are in a position to study about it. For an anisotropic compact star, all the energy conditions like, Weak Energy Condition (WEC), Null Energy Condition (NEC), Strong Energy Condition (SEC) and dominant energy conditions (DEC) are satisfied if and only if the following inequalities hold simultaneously for every points inside the stellar configuration.
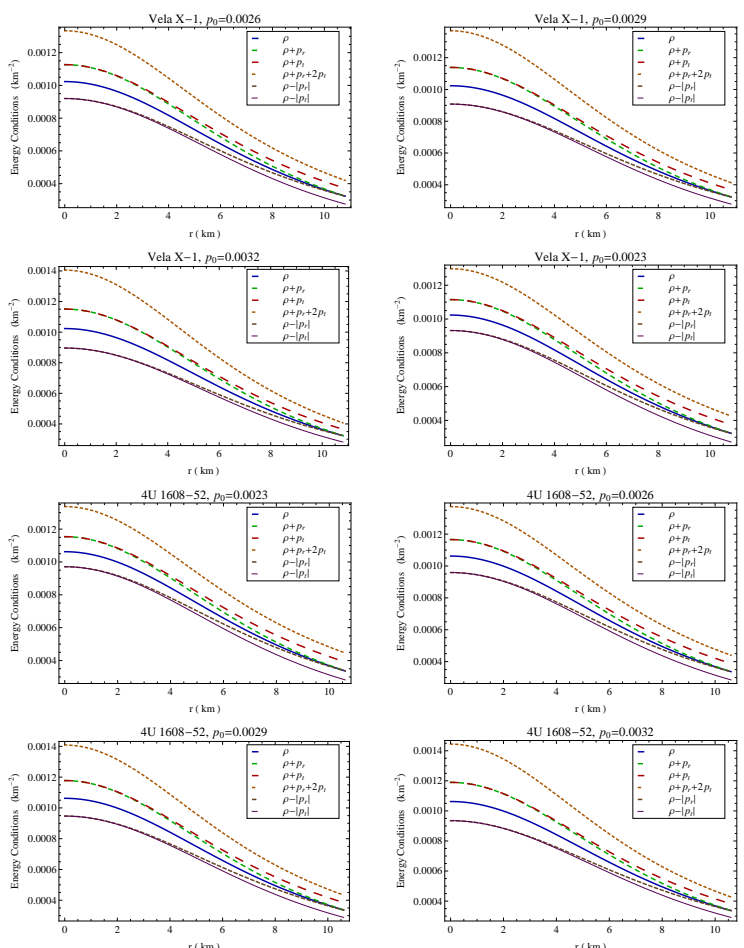

FIG. 7: NEC, WEC, SEC and DEC are plotted against the radius $r$ for a possible modelling of the compact star

Vela X-1 and $4 \mathrm{U} 1608-52$ for different values of $p_{0}$ mentioned in the figures.

$$
\begin{aligned}
W E C: T_{\mu \nu} t^{\mu} t^{\nu} & \geq 0 \text { or } \rho \geq 0, \rho+p_{i} \geq 0 \\
N E C & : T_{\mu \nu} l^{\mu} l^{\nu} \geq 0 \text { or } \rho+p_{i} \geq 0 \\
D E C & : T_{\mu \nu} t^{\mu} t^{\nu} \geq 0 \text { or } \rho \geq\left|p_{i}\right| \\
S E C & : T_{\mu \nu} t^{\mu} t^{\nu}-\frac{1}{2} T_{\lambda}^{\lambda} t^{\sigma} t_{\sigma} \geq 0 \text { or } \rho+\sum_{i} p_{i} \geq 0
\end{aligned}
$$


where $i$ takes the value $r$ and $t$ for radial and transverse pressure. $t^{\mu}$ and $l^{\mu}$ are time-like vector and null vector respectively and $T^{\mu \nu} t_{\mu}$ is nonspace-like vector. To check all the inequality stated above we have drawn the profiles of 1.h.s of (30)-(33) in fig 7 in the interior of the compact star PSR J 1614-2230 and 4U1608-52. The figure shows that all the energy conditions are well satisfied by our present model of compact star.

\section{STABILITY ANALYSIS}

In this section we want to check the stability of the present model with the help of (i) causality condition, (ii) relativistic adiabatic index (iii) Harrison-ZeldovichNovikov's stability condition and (iv) tov equation.

\section{A. Causality Condition}

For a compact star model, the radial and transverse velocity of sound is obtained as,

$$
V_{r}^{2}=\frac{d p_{r}}{d \rho}, \quad V_{t}^{2}=\frac{d p_{t}}{d \rho}
$$

Using the above formulae, for our present model, the

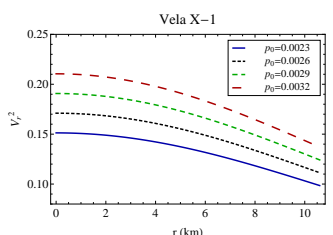

Vela X-1

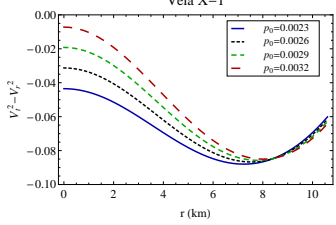

$4 \mathrm{U} 1608-52$

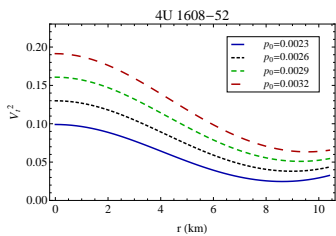

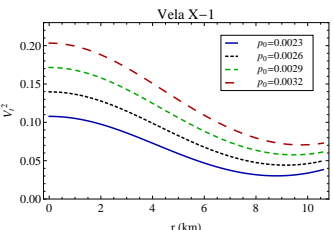

$4 \mathrm{U} 1608-52$

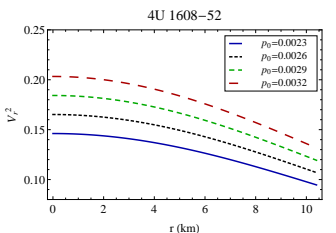

$4 \mathrm{U} 1608-52$

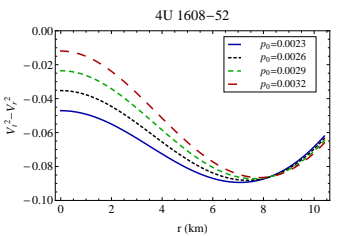

FIG. 8: $V_{r}^{2}, V_{t}^{2}$ and $V_{t}^{2}-V_{r}^{2}$ are plotted against $r$ for $p_{0}=0.0023, p_{0}=0.0026, p_{0}=0.0029$ and $p_{0}=0.0032$ respectively for different values of ' $a$ ' and ' $b$ ' mentioned in Table II for a possible modelling of the compact stars Vela X-1 and 4U 1608-52. radial and transverse velocity of sound are calculated as,

$$
\begin{aligned}
V_{r}^{2}= & \frac{p_{0} \Psi}{\zeta}\left(2 b r^{2}+a\left(2-b r^{4}\right)\right) \\
V_{t}^{2}= & \frac{1}{4 \Psi \zeta}\left[C_{3}-2 C_{4} r^{2}+C_{5} r^{4}+4 C_{6} r^{6}+C_{7} r^{8}\right. \\
& \left.-C_{8} r^{10}+C_{9} r^{12}+C_{10} r^{14}+C_{11} r^{16}\right]
\end{aligned}
$$

where

$$
\zeta=5 a^{2}-5 b+C_{1} r^{2}+C_{2} r^{4}+3 a b^{2} r^{6}+b^{3} r^{8},
$$

and $C_{i}^{\prime} s$ are constants given by,

$$
\begin{aligned}
C_{1}= & a\left(a^{2}+13 b\right) \\
C_{2}= & 3 b\left(a^{2}+4 b\right) \\
C_{3}= & -3 a^{2}+16 a p_{0}-p_{0}^{2} \\
C_{4}= & a^{3}+8 a b+3 a^{2} p_{0}-14 b p_{0}-2 a p_{0}^{2} \\
C_{5}= & a^{4}-19 a^{2} b-15 b^{2}+a\left(17 a^{2}-58 b\right) p_{0} \\
& +2\left(a^{2}+b\right) p_{0}^{2} \\
C_{6}= & -8 a b^{2}+\left(a^{4}+10 a^{2} b-9 b^{2}\right) p_{0}+a\left(-a^{2}+b\right) p_{0}^{2}, \\
C_{7}= & a^{5} p_{0}+8 a^{3} b p_{0}+49 a b^{2} p_{0}+3 b^{2}\left(-5 b+p_{0}^{2}\right) \\
& -a^{4}\left(b+p_{0}^{2}\right)-2 a^{2} b\left(2 b+3 p_{0}^{2}\right) \\
C_{8}= & 4 b\left(a b\left(a^{2}+b\right)-\left(a^{4}+a^{2} b+2 b^{2}\right) p_{0}+a b p_{0}^{2}\right) \\
C_{9}= & b^{2}\left(-b+a p_{0}\right)\left(b+a\left(6 a+p_{0}\right)\right) \\
C_{10}= & 4 a b^{3}\left(-b+a p_{0}\right) \\
C_{11}= & b^{4}\left(-b+a p_{0}\right) .
\end{aligned}
$$

Now at the point $r=0$, the square of radial and transverse velocity of sound are obtained as,

$$
V_{r}^{2}=\frac{3 a p_{0}}{5\left(a^{2}-b\right)}, \quad V_{t}^{2}=-\frac{3 a^{2}-20 a p_{0}+p_{0}^{2}}{20\left(a^{2}-b\right)} .
$$

Moreover, at the center of the star,

$$
V_{t}^{2}-V_{r}^{2}=-\frac{3 a^{2}-8 a p_{0}+p_{0}^{2}}{20\left(a^{2}-b\right)} .
$$

Now by using Le Chatelier's principle we have the speed of sound must be positive inside the stellar interior, i.e., $0<\frac{p_{r}^{\prime}}{\rho^{\prime}}, \frac{p_{t}^{\prime}}{\rho^{\prime}}$. We also know that for a physically acceptable model, the velocity of the sound (both radial and transverse) should be less than the speed of the light i.e., both $\frac{p_{r}^{\prime}}{\rho^{\prime}}, \frac{p_{t}^{\prime}}{\rho^{\prime}}<1$ which is known as the causality condition. Here $\rho^{\prime}$ and $p_{r}^{\prime}$ represent differentiation with respect to $\mathrm{r}$. Combining the above two inequalities we have, $0 \leq V_{r}^{2}, V_{t}^{2} \leq 1$

To find a potentially stable region, in 1992, Herrera proposed a method which is known as "cracking method". This method tells us that a stellar model will be potentially stable if the square of radial velocity of sound exceeds the square of transverse velocity of sound everywhere within the stellar model, otherwise the stellar model will be potentially unstable i.e.,

$$
V_{t}^{2}-V_{r}^{2} \begin{cases}<0 \text { for } 0 \leq r \leq R \Rightarrow & \text { potentially stable } \\ >0 \text { for } 0 \leq r \leq R \Rightarrow & \text { unstable. }\end{cases}
$$


Now at the center of the star, $V_{t}^{2}-V_{r}^{2}<0$ gives,

$$
-\frac{3 a^{2}-8 a p_{0}+p_{0}^{2}}{20\left(a^{2}-b\right)}<0
$$

Using, Eq. (27) from Eq. (37), we further obtain the following inequality:

$$
3 a^{2}-8 a p_{0}+p_{0}^{2}>0 .
$$

The above inequality gives,

$$
p_{0} \notin(0.3944 a, 7.6055 a) .
$$

Combining (28) and (38), we further get,

$$
p_{0} \in(0.18975 a, 0.3944 a) \cup(7.6055 a, 15.8102 a) .(39)
$$

Hence we have obtained a range for $p_{0}$ that could describe a physically reasonable stellar structure.

\section{B. Relativistic Adiabatic index}

The adiabatic index determines the stability of a compact object and for an anisotropic stellar configuration it is defined as,

$$
\Gamma_{r}=\frac{\rho(r)+p_{r}(r)}{p_{r}(r)} \frac{d p_{r}(r)}{d \rho(r)} .
$$
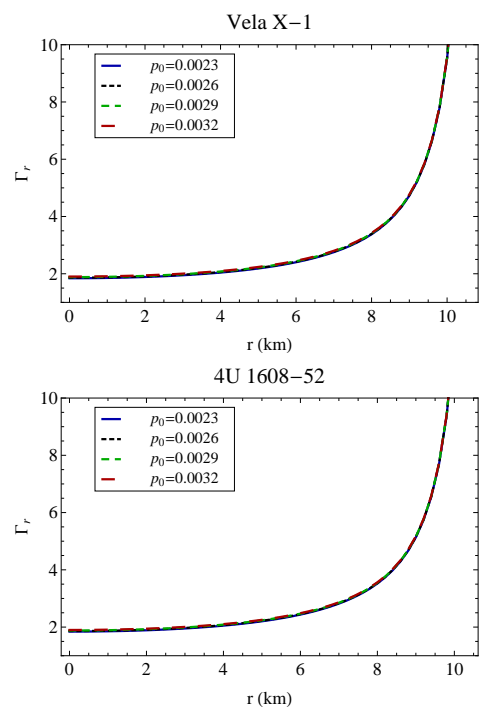

FIG. 9: Adiabatic Index is plotted against $r$ inside the stellar interior for a possible modelling of the compact star Vela X-1 and 4U 1608-52 for different values of $p_{0}$ mentioned in the figures.

For our model we have

$$
\Gamma_{r}=\frac{3 a+p_{0}+\left(a^{2}+5 b\right) r^{2}+D_{1} r^{4}+D_{2} r^{6}}{p_{0}\left(1-a r^{2}\right) \Psi} V_{r}^{2},
$$

where $D_{1}$ and $D_{2}$ are constants given by,

$$
D_{1}=2 a b-a^{2} p_{0}+b p_{0} ; \quad D_{2}=b\left(b-a p_{0}\right) .
$$

Any stellar configuration will maintain its stability if adiabatic index $\Gamma_{r}>4 / 3$ [27]. For our solution, the adiabatic index $\Gamma_{r}$ takes the value more than $4 / 3$ throughout the interior of the compact star, as evident from Fig. 9.

\section{Tov equation}

Now we are ready to check the stability of our present model under three different forces viz gravitational force $F_{g}$, hydrostatics force $F_{h}$ and anisotropic force $F_{a}$. The effect of the above three forces can be described by the conservation equation given by

$$
\nabla^{\mu} T_{\mu \nu}=0
$$

known as TOV equation. Now using the expression given in (1) into (41) one can obtain the following equation:

$$
-\frac{\nu^{\prime}}{2}\left(\rho+p_{r}\right)+\frac{2}{r}\left(p_{t}-p_{r}\right)=p_{r}^{\prime} .
$$

The eqn. (42) can be written as,

$$
F_{g}+F_{h}+F_{a}=0,
$$
as:

where the expression for $F_{g}, F_{h}$ and $F_{a}$ are obtained

$$
\begin{aligned}
F_{g}= & -\frac{\nu^{\prime}}{2}\left(\rho+p_{r}\right) \\
= & \frac{r}{2 \kappa \Psi^{3}}\left\{\left(a+p_{0}+\left(a^{2}+b-a p_{0}\right) r^{2}+2 a b r^{4}\right.\right. \\
& \left.+b^{2} r^{6}\right) \times\left(3 a+p_{0}+\left(a^{2}+5 b-a p_{0}\right) r^{2}\right. \\
& \left.\left.+2 a b r^{4}+b^{2} r^{6}\right)\right\} \\
F_{h}= & -\frac{d p_{r}}{d r}=\frac{2 p_{0}}{\kappa \Psi^{2}}\left(2 b r^{3}+a r\left(2-b r^{4}\right)\right) \\
F_{a}= & \frac{2 \Delta}{r}=\frac{r}{8 \kappa \Psi^{3}}\left[A_{1}+A_{2} r^{2}+A_{3} r^{4}+A_{4} r^{6}+A_{5} r^{8}\right. \\
& \left.+4 a b^{3} r^{10}+b^{4} r^{12}\right] .
\end{aligned}
$$

The three different forces acting on the system are shown in fig. 10 for the compact stars Vela X-1 and 4U 1608-52 for different values of $p_{0}$.

\section{Harrison-Zeldovich-Novikov's stability condition}

Depending on the mass and central density of the star, Harrison et al. [30] and Zeldovich-Novikov [131] proposed the stability condition for the model of compact star. From their investigation they suggested that for stable configuration $\frac{\partial M}{\partial \rho_{c}}>0$, where $M, \rho_{c}$ denotes the mass and central density of the compact star. 
TABLE III: The numerical values of central pressure $\left(p_{c}\right)$ in dyne $\cdot \mathrm{cm}^{-2}$ unit, surface anisotropy in dyne $\cdot \mathrm{cm}^{-2}$ unit, central radial velocity $\left(V_{r}(0)\right)$, surface radial velocity $\left(V_{r}(R)\right)$, central transverse velocity $\left(V_{t}(0)\right)$, surface transverse velocity $\left(V_{t}(R)\right)$ for a possible modelling of the compact star $4 \mathrm{U} 1608-52[26]$.

\begin{tabular}{lccccccc}
\hline$p_{0}$ & $p_{c}$ & $\Delta_{s}=p_{t}(R)$ & $V_{r}(0)$ & $V_{r}(R)$ & $V_{t}(0)$ & $V_{t}(R)$ & $\Gamma_{r}(0)$ \\
\hline 0.0023 & $1.11134 \times 10^{35}$ & $6.64583 \times 10^{34}$ & 0.382269 & 0.305075 & 0.314703 & 0.185608 & 1.84249 \\
0.0026 & $1.2563 \times 10^{35}$ & $6.26024 \times 10^{34}$ & 0.406435 & 0.324362 & 0.360459 & 0.212585 & 1.86155 \\
0.0029 & $1.40126 \times 10^{35}$ & $5.87465 \times 10^{34}$ & 0.429243 & 0.342564 & 0.400895 & 0.236505 & 1.88061 \\
0.0032 & $1.54621 \times 10^{35}$ & $5.48905 \times 10^{34}$ & 0.450899 & 0.359847 & 0.437487 & 0.258218 & 1.89967 \\
\hline
\end{tabular}
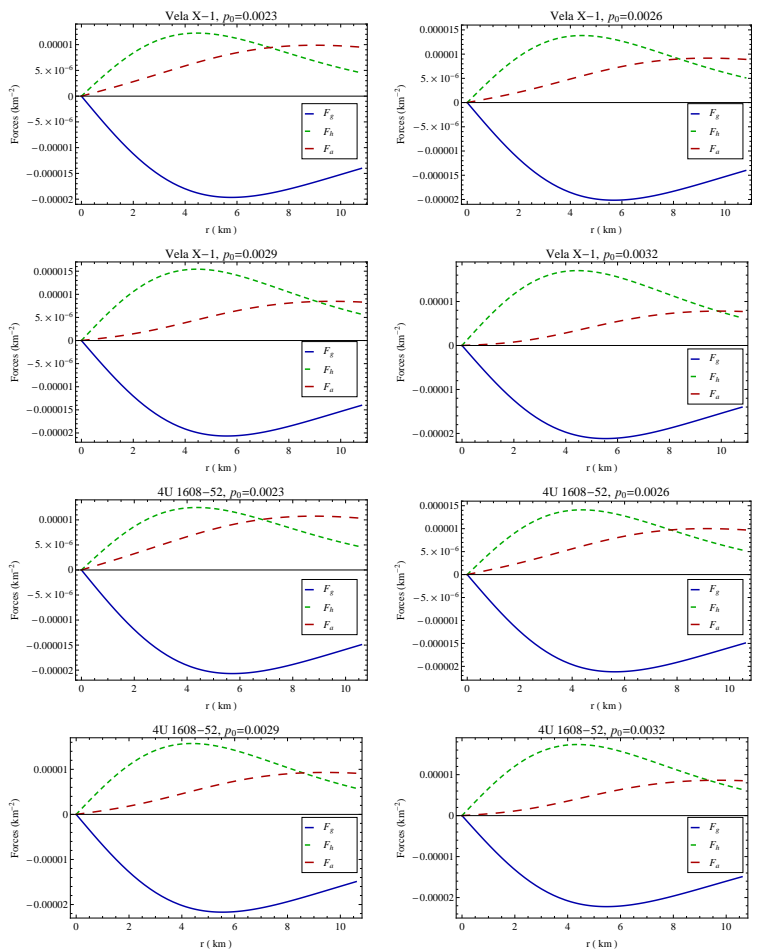

FIG. 10: Different forces are plotted against radial parameter $r$ for a possible modelling of the compact star Vela X-1 and $4 \mathrm{U} 1608-52$ for different values of $p_{0}$ mentioned in the figures.
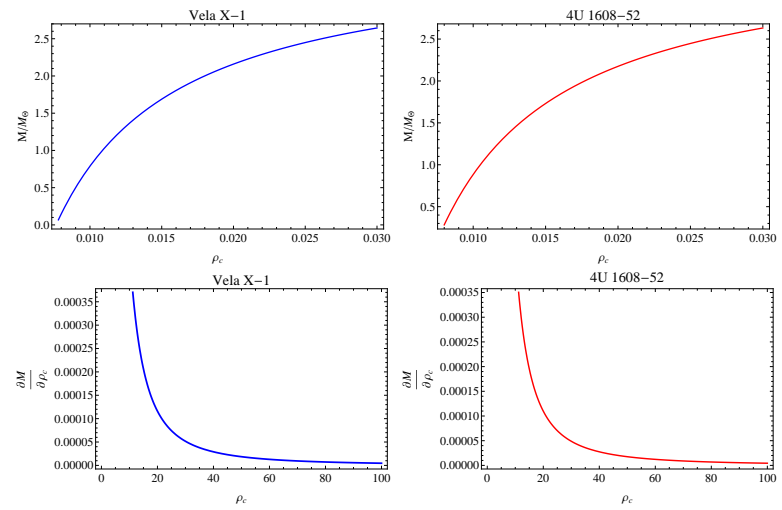

FIG. 11: Variation of mass $\left(\mathrm{M} / M_{\odot}\right)$ and $\frac{\partial M}{\partial \rho_{c}}$ with respect to the central density $\rho_{c}$ for a possible modelling of the compact star Vela X-1 and 4U 1608-52.
For our present model,

$$
\frac{\partial M}{\partial \rho_{c}}=\frac{12 \pi R^{3}\left(1+b\left(R^{4}-R^{6}\right)\right)}{\left(3+3 b R^{4}+8 \pi R^{2} \rho_{c}\right)^{2}} .
$$

Above expression of $\frac{\partial M}{\partial \rho_{c}}$ is positive and hence the stability condition is well satisfied. The variation of the mass function as well as $\frac{\partial M}{\partial \rho_{c}}$ with respect to the central density is depicted in fig. 11 .

\section{E. Linearized stability analysis}

We have already matched the interior space-time continuously to an exterior schwarzschild vacuum solution with $p_{r}=0$ at the junction interface $\Sigma$, with junction radius $R$.

Now by using the standard Darmois-Israel formalism $[42,43]$ and with the help of Lanczos equations the surface stresses can be obtained, as follows :

$$
\begin{aligned}
\sigma= & -\frac{1}{4 \pi R}\left[\sqrt{1-\frac{2 M}{R}+\dot{R}^{2}}\right. \\
& \left.-\sqrt{\frac{1}{\left(1+a R^{2}+b R^{4}\right)}+\dot{R}^{2}}\right],
\end{aligned}
$$

and

$$
\begin{aligned}
\mathcal{P}= & \frac{1}{8 \pi R}\left[\frac{1-\frac{M}{R}+\dot{R}+R \ddot{R}}{\sqrt{1-\frac{2 M}{R}+\dot{R}^{2}}}\right. \\
& \left.-\frac{\left(1+a R^{2}+b R^{4}\right)^{-1}+\dot{R}^{2} \frac{1-b R^{4}}{1+a R^{2}+b R^{4}}+R \ddot{R}}{\sqrt{\left(1+a R^{2}+b R^{4}\right)^{-1}+\dot{R}^{2}}}\right]
\end{aligned}
$$

Where $\sigma$ and $\mathcal{P}$ represent the surface density and tangential surface pressure of the internal forces on the junction surface.

We now proceed with the surface mass of the thin shell is given by

$$
m_{s}=4 \pi R^{2} \sigma .
$$

When one substitutes Eq. (48) into Eq. (50), one can get

$m_{s}=-R\left[\sqrt{1-\frac{2 M}{R}+\dot{R}^{2}}-\sqrt{\dot{R}^{2}+\left(1+a R^{2}+b R^{4}\right)^{-1}}\right]$, 
TABLE IV: The numerical values of central pressure $\left(p_{c}\right)$ in dyne $\cdot \mathrm{cm}^{-2}$ unit, surface anisotropy in dyne $\cdot \mathrm{cm}^{-2}$ unit, central radial velocity $\left(V_{r}(0)\right)$, surface radial velocity $\left(V_{r}(R)\right)$, central transverse velocity $\left(V_{t}(0)\right)$, surface transverse velocity $\left(V_{t}(R)\right)$ for a possible modelling of the compact star Vela X-1 [89].

\begin{tabular}{|c|c|c|c|c|c|c|c|}
\hline$p_{0}$ & $p_{c}$ & $\Delta_{s}=p_{t}(R)$ & $V_{r}(0)$ & $V_{r}(R)$ & $V_{t}(0)$ & $V_{t}(R)$ & $\Gamma_{r}(0)$ \\
\hline 0.0023 & $1.11134 \times 10^{35}$ & $6.2321 \times 10^{34}$ & 0.38895 & 0.311366 & 0.328293 & 0.200689 & 1.84302 \\
\hline 0.0026 & $1.2563 \times 10^{35}$ & $5.84535 \times 10^{34}$ & 0.413539 & 0.33105 & 0.373797 & 0.227046 & 1.86275 \\
\hline 0.0029 & $1.40126 \times 10^{35}$ & $5.45861 \times 10^{34}$ & 0.436746 & 0.349628 & 0.414194 & 0.250647 & 1.88248 \\
\hline 0.0032 & $1.54621 \times 10^{35}$ & $5.07186 \times 10^{34}$ & 0.45878 & 0.367267 & 0.450859 & 0.272209 & 1.90222 \\
\hline
\end{tabular}

Now, differentiating twice the expression in (50), and taking into account the radial derivative of $\sigma^{\prime}$, we can obtain,

$$
\left(\frac{m_{s}}{2 R}\right)^{\prime \prime}=\Upsilon-4 \pi \sigma^{\prime} \eta,
$$

where the parameters $\eta$ and $\Upsilon$ are given by,

$$
\eta=\frac{\mathcal{P}^{\prime}}{\sigma^{\prime}}, \quad \Upsilon=\frac{4 \pi}{R}(\sigma+\mathcal{P})+2 \pi R \Xi^{\prime} .
$$

The expression found above will play an important role in stability analysis of static solutions. Here the parameter $\eta$ is used to determine the stability of the system. $\eta$ can be interpreted as the square of velocity of sound on the shell and it lies in the range $0<\eta \leq 1$. For our present model,

$$
\Xi=\frac{a R+2 b R^{3}}{4 \pi+4 a \pi R^{2}+4 b \pi R^{4}} \sqrt{\dot{R}^{2}+\left(1+a R^{2}+b R^{4}\right)^{-1}} .
$$

Now we are going to check for the stability analysis of the solution. In order to study the dynamical stability of the stellar model we consider a linear perturbation around those static solutions.

Rearranging (48), we get,

$$
\dot{R}^{2}+V(R)=0,
$$

with $V(R)$ given by

$V(R)=1-\frac{m(R)+M}{R}-\left(\frac{m_{s}(R)}{2 R}\right)^{2}-\left(\frac{M-m(R)}{m_{s}(R)}\right)^{2}$.

Note that the potential function $V(R)$ helps us to determine the stability region for the thin shell under the linear perturbation. By considering the Taylor series expansion around the static solution $R_{0}$, up to second order we get,

$$
\begin{aligned}
V(R)= & V\left(R_{0}\right)+\left(R-R_{0}\right) V^{\prime}\left(R_{0}\right)+\frac{\left(R-R_{0}\right)^{2}}{2} V^{\prime \prime}\left(R_{0}\right) \\
& +\mathcal{O}\left[\left(R-R_{0}\right)^{3}\right],
\end{aligned}
$$

where prime corresponding to a derivative with respect to R. According to the standard method we are linearizing around the static radius $R=R_{0}$, we must have $V\left(R_{0}\right)=$ 0 and $V^{\prime}\left(R_{0}\right)=0$. Now, $V^{\prime}\left(R_{0}\right)=0$ gives the following relation

$$
\begin{aligned}
\left(\frac{m_{s}\left(R_{0}\right)}{2 R_{0}}\right)^{\prime}=\Phi= & \frac{R_{0}}{m_{s}\left(R_{0}\right)}\left[-\left(\frac{m\left(R_{0}\right)+M}{R_{0}}\right)^{\prime}\right. \\
& \left.-2\left(\frac{M-m\left(R_{0}\right)}{m_{s}\left(R_{0}\right)}\right)\left(\frac{M-m\left(R_{0}\right)}{m_{s}\left(R_{0}\right)}\right)^{\prime}\right]
\end{aligned}
$$

With this definition the second derivative $V^{\prime \prime}\left(R_{0}\right)$ can be written as

$$
\begin{aligned}
V^{\prime \prime}\left(R_{0}\right)= & -\left(\frac{m+M}{R_{0}}\right)^{\prime \prime}-2\left[\left(\frac{m_{s}}{2 R_{0}}\right)^{\prime}\right]^{2} \\
& -2\left(\frac{m_{s}}{2 R_{0}}\right)\left(\frac{m_{s}}{2 R_{0}}\right)^{\prime \prime}-2\left[\left(\frac{M-m}{m_{s}}\right)^{\prime}\right]^{2} \\
& -2\left(\frac{M-m}{m_{s}}\right)\left(\frac{M-m}{m_{s}}\right)^{\prime \prime}
\end{aligned}
$$

Thus, for a static configuration $V\left(R_{0}\right)=0, V^{\prime}\left(R_{0}\right)=0$. Now from (54) and (56), we get,

$$
\dot{R}^{2}+\frac{1}{2} V^{\prime \prime}\left(R_{0}\right)\left(R-R_{0}\right)^{2}+\mathcal{O}\left[\left(R-R_{0}\right)^{3}\right]=0,
$$

To ensure the stability of static configuration at $\mathrm{R}=R_{0}$, we must have, $V^{\prime \prime}\left(R_{0}\right)>0$. For the sake of simplicity we rearrange the Eq. (59), which turns out

$$
\Rightarrow V^{\prime \prime}=\Pi-2 \Phi^{2}-\left.\frac{m_{s}}{R_{0}}\left(\Upsilon-4 \pi \sigma^{\prime} \eta\right)\right|_{R_{0}} .
$$

where,

$$
\begin{aligned}
\Pi= & -\left(\frac{m+M}{R_{0}}\right)^{\prime \prime}-2\left[\left(\frac{M-m}{m_{s}}\right)^{\prime}\right]^{2} \\
& -2\left(\frac{M-m}{m_{s}}\right)\left(\frac{M-m}{m_{s}}\right)^{\prime \prime} .
\end{aligned}
$$

(For details calculations please refer ref [2].)

Now by assuming $\eta\left(R_{0}\right)=\eta_{0}$ and using Eq. (60) for $V^{\prime \prime}\left(R_{0}\right)>0$ we have

$$
\left.\eta_{0} \frac{d \sigma^{2}}{d R}\right|_{R_{0}}>\frac{\sigma}{2 \pi}\left[\Upsilon+\frac{R_{0}}{m_{s}}\left(2 \Phi^{2}-\Pi\right)\right]=\Omega \text { (say). }
$$


From the above inequality we get the following two cases,

$$
\begin{aligned}
& \eta_{0}>\Omega\left(\left.\frac{d \sigma^{2}}{d R}\right|_{R_{0}}\right)^{-1}, \text { if }\left.\frac{d \sigma^{2}}{d R}\right|_{R_{0}}>0, \\
& \eta_{0}<\Omega\left(\left.\frac{d \sigma^{2}}{d R}\right|_{R_{0}}\right)^{-1}, \text { if }\left.\frac{d \sigma^{2}}{d R}\right|_{R_{0}}<0 .
\end{aligned}
$$

Now to make a comment regarding the stability region of the stellar configuration, the profile of $\frac{d \sigma_{0}^{2}}{d R_{0}}$ is plotted in fig 12 . So, we can conclude that the stability region is given by eq. (64) since $\frac{d \sigma_{0}^{2}}{d R_{0}}<0$ for our present paper.

\section{CALCULATION OF TIDAL LOVE NUMBER OF COMPACT NEUTRON STAR WITH ANISOTROPIC PRESSURE}

Gravitational perturbation are very much important, though very much difficult to handle, since they convey information about the structure of the gravitational field equations. So let's start by trying to answer these question: Given a metric for instance Schwarzschild geometry or metric of a compact neutron star, how does a small perturburtation in the metric evolve? Here small perturbation means anything- a wave, a celestial body, a falling particle - that disturb the background metric slightly. Mathematically consider a background metric ${ }^{(0)} g_{\mu \nu}\left(x^{\nu}\right)$ - metric of a neutron star. With small perturbation $h_{\mu \nu}\left(x^{\nu}\right)$, the modified new metric can be written as

$$
g_{\mu \nu}\left(x^{\nu}\right)={ }^{(0)} g_{\mu \nu}\left(x^{\nu}\right)+h_{\mu \nu}\left(x^{\nu}\right),
$$

where the background geometry of spacetime of a spherical static star can be written as

$$
\begin{aligned}
{ }^{(0)} d s^{2} & ={ }^{(0)} g_{\mu \nu} d x^{\mu} d x^{\nu} \\
& =-e^{\nu(r)} d t^{2}+e^{\lambda(r)} d r^{2}+r^{2}\left(d \theta^{2}+\sin ^{2} \theta d \phi^{2}\right) .
\end{aligned}
$$

For the linearized metric perturbation $h_{\alpha \beta}$, using the method as in [90], [6], we restrict ourselves to static $l=2, m=0$ even parity perturbation. With these assumptions the perturbed metric becomes

$h_{\alpha \beta}=\operatorname{diag}\left[H_{0}(r) e^{\nu}, H_{2}(r) e^{\lambda}, r^{2} K(r), r^{2} \sin ^{2} \theta K(r)\right] Y_{2 m}(\theta, \phi)$,

Because of these external perturbation the equalibrium configuration of a star gets deformed tidally. As a cosequence spherically symmetric star develpes a quadrapole moment $Q_{i j}$. And $Q_{i j}$ can be related with the linear order external tidal field $\varepsilon_{i j}$ as [40]

$$
Q_{i j}=-\Lambda \varepsilon_{i j},
$$

where $\Lambda$ is the tidal deformability of the neutron star and it is related to the tidal love number $k_{2}$ as $\Lambda=$ $\frac{2}{3} k_{2} R^{5}[40]$.

Now for the spherically static metic (66), the stressenergy tensor is given as

$$
{ }^{(0)} T_{\chi}^{\xi}=\left(\rho+p_{t}\right) u^{\xi} u_{\chi}+p_{t} g_{\chi}^{\xi}+\left(p_{r}-p_{t}\right) \eta^{\xi} \eta_{\chi} .
$$

Furthermore the energy momentum tensor is perturbed by a perturbation tensor $\delta T_{\chi}^{\xi}$. The perturbed tensor is defined by

$$
T_{\chi}^{\xi}={ }^{(0)} T_{\chi}^{\xi}+\delta T_{\chi}^{\xi}
$$

where the non-zero components of $T_{\chi}^{\xi}$ are

$$
\begin{aligned}
T_{t}^{t} & =-\frac{d \rho}{d p_{r}} \delta p_{r}(r) Y(\theta, \phi)-\rho(r), \\
T_{r}^{r} & =\delta p_{r}(r) Y(\theta, \phi)+p_{r}(r), \\
T_{\theta}^{\theta} & =\frac{d p_{t}}{d p_{r}} \delta p_{r}(r) Y(\theta, \phi)+p_{t}(r), \\
T_{\phi}^{\phi} & =\frac{d p_{t}}{d p_{r}} \delta p_{r}(r) Y(\theta, \phi)+p_{t}(r) .
\end{aligned}
$$

Note that in Eq. (71)-(74), and hereafter, we use $p \equiv p_{r}$ to denote the radial pressure. With these perturbed quantities we can write down the perturbed Einstein Field Equations

$$
G_{\chi}^{\xi}=8 \pi T_{\chi}^{\xi}
$$

where the Einstein tensor $G_{\chi}^{\xi}$ is calculated using the metric $g_{\chi \xi}$.

From the various components of background Einstein field equation ${ }^{(0)} G_{\chi}^{\xi}=8 \pi^{(0)} T_{\chi}^{\xi}$, we can have the following things (which will be used later on):

$$
\begin{aligned}
{ }^{(0)} G_{t}^{t} & =8 \pi^{(0)} T_{t}^{t} \\
\Rightarrow \lambda^{\prime}(r) & =\frac{8 \pi r^{2} e^{\lambda(r)} \rho(r)-e^{\lambda(r)}+1}{r}, \\
{ }^{(0)} G_{r}^{r} & =8 \pi^{(0)} T_{r}^{r} \\
\Rightarrow \nu^{\prime}(r) & =\frac{8 \pi r^{2} p_{r}(r) e^{\lambda(r)}+e^{\lambda(r)}-1}{r} .
\end{aligned}
$$

Also we know that $\nabla_{\xi}^{(0)} T_{\chi}^{\xi}=0$. Choosing $\xi=r$, by expanding and solving the equation, we can find the expression as 

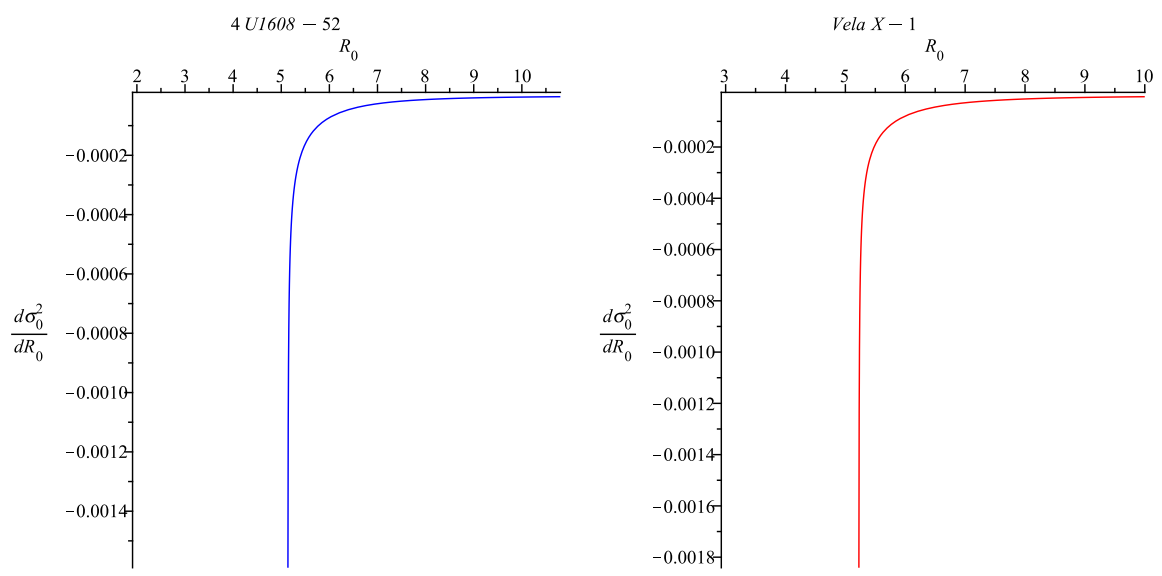

FIG. 12: $\frac{d \sigma_{0}^{2}}{d R_{0}}$ has been shown against $R_{0}$

$p_{r}^{\prime}(r)=\frac{1}{2 r}\left[-4 p_{r}(r)+4 p_{t}(r)-r p_{r}(r) \nu^{\prime}(r)-r \rho(r) \nu^{\prime}(r)\right]$.

Now from the various components of perturbed Einstein equation (75), we get the following relations

$G_{\theta}^{\theta}-G_{\phi}^{\phi}=0 \Rightarrow H_{0}(r)=H_{2}(r)=H(r)$,

$G_{r}^{\theta}=0 \Rightarrow K^{\prime}=H^{\prime}+H \nu^{\prime}$

$G_{\theta}^{\theta}+G_{\phi}^{\phi}=8 \pi\left(T_{\theta}^{\theta}+T_{\phi}^{\phi}\right) \Rightarrow \delta p=\frac{H(r) e^{-\lambda(r)}\left(\lambda^{\prime}(r)+\nu^{\prime}(r)\right)}{16 \pi r \frac{d p_{t}}{d p_{r}}}$.
Using the identity

$\frac{\partial^{2} Y(\theta, \phi)}{\partial \theta^{2}}+\cot (\theta) \frac{\partial Y(\theta, \phi)}{\partial \theta}+\csc ^{2}(\theta) \frac{\partial^{2} Y(\theta, \phi)}{\partial \phi^{2}}=-6 Y(\theta, \phi)$ , eqn $(76),(77),(78),(80),(81)$ we have the master equation for $H(r)$ as

$$
\begin{aligned}
& -\frac{1}{e^{-\lambda(r)} Y(\theta, \phi)}\left[G_{t}^{t}-G_{r}^{r}\right]=-\frac{8 \pi}{e^{-\lambda(r)} Y(\theta, \phi)}\left[T_{t}^{t}-T_{r}^{r}\right] \\
& \Rightarrow H^{\prime \prime}(r)+\mathcal{R} H^{\prime}(r)+\mathcal{S} H(r)=0
\end{aligned}
$$

Where,

$$
\mathcal{R}=-\left[\frac{-e^{\lambda(r)}-1}{r}-4 \pi r e^{\lambda(r)}\left(p_{r}-\rho(r)\right)\right]
$$

$$
\begin{array}{r}
\mathcal{S}=-\left[1 6 \pi e ^ { \lambda ( r ) } \left(p_{r}\left(e^{\lambda(r)}-2\right)-p_{t}(r)\right.\right. \\
-\rho(r))+64 \pi^{2} r^{2} p_{r}^{2} e^{2 \lambda(r)}+\frac{4 e^{\lambda(r)}+e^{2 \lambda(r)}+1}{r^{2}} \\
\left.+\frac{-4 \pi \frac{d \rho}{d p_{r}} e^{\lambda(r)}\left(p_{r}+\rho(r)\right)-4 \pi e^{\lambda(r)}\left(p_{r}+\rho(r)\right)}{\frac{d p_{t}}{d p_{r}}}\right]
\end{array}
$$

$$
e^{\lambda(r)}=1+a r^{2}+b r^{4}, a=1 / R^{2}, b=\text { tively. }
$$

$\frac{1}{R^{4}}\left[\left(1-\frac{2 M}{R}\right)^{-1}-2\right] . \quad M \& R$ are mass and radius of the star respectively. And the expression for $p_{r}, p_{t} \& \rho$ are given in the equation $(7),(8),(11)$ respec-
Outside the star, considering Schwarzschild metric and by setting $\rho=0, p_{r}=0, p_{t}=0, e^{\lambda}=1 /(1-2 M / r)$, equation (82) becomes:

$$
-H^{\prime \prime}(r)+\left(\frac{1}{2 M-r}-\frac{1}{r}\right) H^{\prime}(r)+\frac{2 H(r)\left(2 M^{2}-6 M r+3 r^{2}\right)}{r^{2}(r-2 M)^{2}}=0
$$


$H(r)=\frac{3 c_{1} r(2 M-r)}{M^{2}}+\frac{c_{2}\left(-2 M\left(2 M^{3}+4 M^{2} r-9 M r^{2}+3 r^{3}\right)-3 r^{2}(r-2 M)^{2} \log \left(\frac{r}{M}-2\right)+3 r^{2}(r-2 M)^{2} \log \left(\frac{r}{M}\right)\right)}{2 M^{2} r(2 M-r)}$

Where $c_{1} \& c_{2}$ are integration constant which yet to be determined. In order to get these constants, let's expand the equation (86).

$$
H(r)=-\frac{3 c_{1} r^{2}}{M^{2}}+\frac{6 c_{1} r}{M}-\frac{c_{2}\left(8 M^{3}\right)}{5 r^{3}}+\mathcal{O}\left(\left(\frac{1}{r}\right)^{4}\right)
$$

Now in the star's local asymptotic rest frame (asymptotically mass-centered Cartesian coordinates) at large $r$ the metric coefficient $g_{t t}$ is given by [118], [40]

$$
\begin{aligned}
\frac{\left(1-g_{t t}\right)}{2}= & -\frac{M}{r}-\frac{3 Q_{i j}}{2 r^{3}}\left(n^{i} n^{j}-\frac{1}{3} \delta^{i j}\right)+\mathcal{O}\left(\frac{1}{r^{3}}\right) \\
& +\frac{1}{2} \mathcal{E}_{i j} x^{i} x^{j}+\mathcal{O}\left(r^{3}\right)
\end{aligned}
$$

where $n^{i}=x^{i} / r$. Matching the asymptotic solution from equation (87) to the expansion from equation (88) and using the equation (68) we have

$$
c_{1}=-\frac{M^{2} \mathcal{E}}{3}, \quad c_{2}=\frac{15 \mathcal{Q}}{8 M^{3}}
$$

We now solve for $k_{2}$ in terms of $H$ and its derivative at the star's surface $r=R$ using equations (89) and (86), and use equation $k_{2}=\frac{3}{2} \Lambda R^{-5}$ to obtain the expression for tidal love number

$$
k_{2}=\left[8(1-2 \mathcal{C})^{2} \mathcal{C}^{5}(2 \mathcal{C}(y-1)-y+2)\right] / X
$$

Where,

$$
\begin{aligned}
X & =5(2 \mathcal{C}(\mathcal{C}(2 \mathcal{C}(\mathcal{C}(2 \mathcal{C}(y+1)+3 y-2)-11 y+13)+3(5 y-8))-3 y+6) \\
& \left.+3(1-2 \mathcal{C})^{2}(2 \mathcal{C}(y-1)-y+2) \log \left(\frac{1}{\mathcal{C}}-2\right)-3(1-2 \mathcal{C})^{2}(2 \mathcal{C}(y-1)-y+2) \log \left(\frac{1}{\mathcal{C}}\right)\right)
\end{aligned}
$$

Here $\mathcal{C}=\frac{M}{R}$ and $y$ depends on $r, H$ and it's derivatives

$$
y=\frac{r H^{\prime}(r)}{H(r)_{R}} .
$$

To get the numerical value of $k_{2}$ for a particular star, first of all we have to find the numerical value of $y$. To do this let's modify master differential equation (82) using the equation (92) as [86]

$$
r y^{\prime}+y^{2}+(r \mathcal{R}-1) y+r^{2} \mathcal{S}=0
$$

At $\mathcal{C} \rightarrow 0$ we expect $k_{2} \rightarrow 0$ and hence $y(0)=2$. Also one can check numerically that at $\mathcal{C}=1 / 2$ the tidal love number vanishes for all value of $y$. It is because the tidal deformability of any order $l$, both electric and magnetic, vanish in the Black hole limit. In other words, the multipolar structure of a black hole is not affected by the tidal field. This can be view as a corollary of the no-hair theorem, and recently it has been proved beyond the perturbative level [25].

After solving the the differential equation (93) using initial value $y(0)=2$, equation (83) \& eqn (84), we can calculate $k_{2}$ from the equation (90).

In the fig. 13 the variation of $y$ w.r.t. $p_{0}$ are shown for four different compact stars. In the figure 14 the variation of tidal love number $k_{2}$ against the central pressure $p_{0}$ are shown for four different compact objects. We found that tidal love number $k_{2}$ decreases monotonically with increasing $p_{0}$. Here the maximum range of $p_{0}$ is $0.3944 a$ as in the equation (39). Where as the minimum range of $p_{0}$ varies for different compact stars. The minimum possible value of $p_{0}$ for some compact star is given in the table V. 

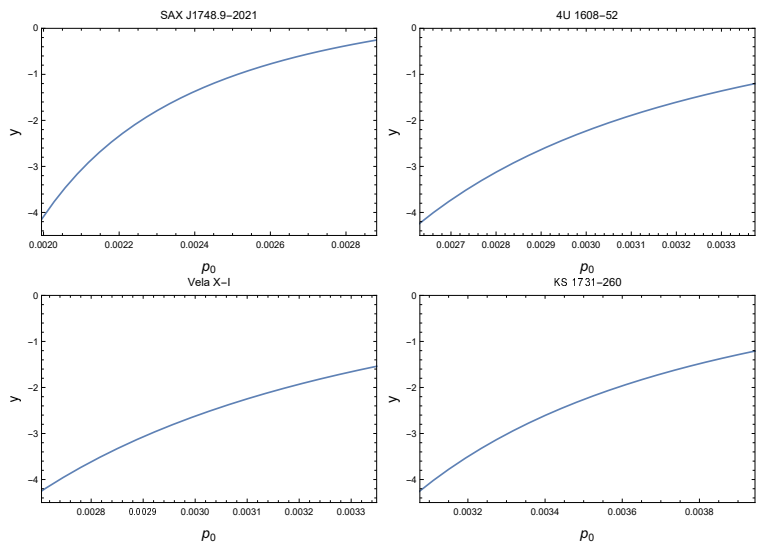

FIG. 13: $y$ is plotted over $p_{0}$ for four different compact star SAXJ1748.9 - 2021, 4U1608 - 52, VelaX - I, $K S 1731-260$. The value of $M \& R$ of these stars are take from the paper [92]. Only allowed range of $p_{0}$ are considered.

TABLE V: The numerical value of Mass, Radius, minimum and maxium range of $p_{0}$ for different compact star. $a=1 / R^{2}$. Numerical values of mass and radius are taken from [92]

\begin{tabular}{|c|c|c|c|c|}
\hline Name & min. $p_{0}$ & max. $p_{0}$ & $\mathrm{M}\left(M_{\odot}\right)$ & $R \mathrm{~km}$ \\
\hline \hline SAX J1748.9-2021 & $0.27305 a$ & $0.3944 a$ & $1.81 \pm 0.25$ & $11.7 \pm 1.7$ \\
\hline 4U 1608-52 & $0.30750 a$ & $0.3944 a$ & $1.74 \pm 0.14$ & $10.811 \pm 0.19$ \\
\hline Vela X-I & $0.318500 a$ & $0.3944 a$ & $1.77 \pm 0.08$ & $10.852 \pm 0.1$ \\
\hline KS 1731-260 & $0.30755 a$ & $0.3944 a$ & $1.61 \pm 0.35$ & $10 \pm 2.2$ \\
\hline
\end{tabular}

\section{DISCUSSION}

In our present paper we proposed a new model of compact star in the background of general theory of relativity. In this work we have focused on the compact object $4 \mathrm{U}$ 1608-52 and Vela X-1 whose estimated masses and radii are obtained in Table I. Our present model satisfies the following conditions:

- Regularity of metric potential: Both the metric potentials $e^{\nu}, e^{\lambda}$ are regular i.e., free from all kinds of singularity inside the stellar interior. $\left.e^{\lambda}\right|_{r=0}=$ $1,\left.e^{\nu}\right|_{r=0}=\exp \left[\frac{2 p_{0}\left(a^{2}+2 b\right)}{b \sqrt{4 b-a^{2}}} \tan ^{-1}\left(\frac{a}{\sqrt{4 b-a^{2}}}\right)+B\right]$.

- The matter density, both radial and transverse pressures take maximum value at the center of the star and gradually decreases towards the boundary, i.e., all are monotonic decreasing functions of $r$ and at the same time they do not suffer from any kinds of singularity. Both the pressures depend on dimensionless quantity $p_{0}$ and the central pressure increases for the increasing values of $p_{0}$.

- The anisotropic factor vanishes at the center of the star and $\Delta>0$ for $0 \leq r \leq R$. For posi-
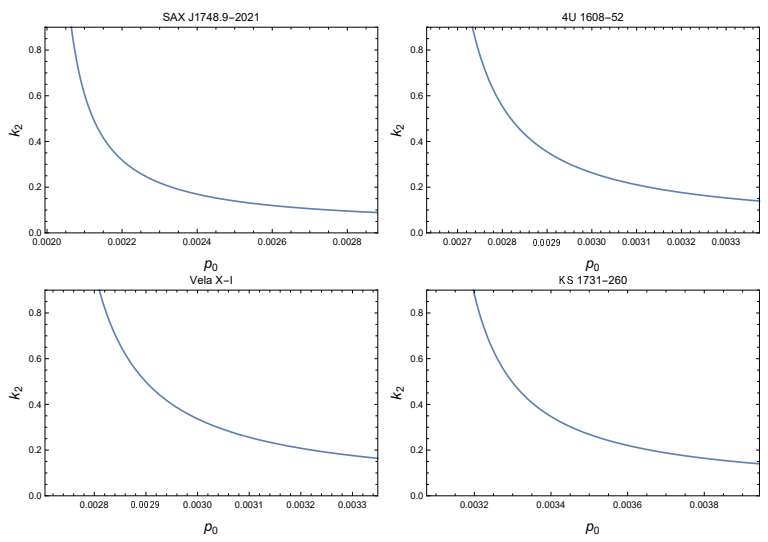

FIG. 14: Tidal love number $k_{2}$ is plotted against $p_{0}$ for compact star $S A X J 1748.9$ - 2021, $4 U 1608$ - 52,

VelaX $-I, K S 1731-260$. The value of $M \& R$ of these stars are take from the paper [92]. Only allowed range of $p_{0}$ are considered.

tive anisotropic factor makes the system more stable and it helps to construct more compact object [21]. The equation of state parameters are plotted against ' $r$ ' inside the stellar interior in fig. 4 and one can note that $0<\omega_{r}, \omega_{t}<1$.

- We have verified that all the energy conditions are satisfied by our model with the help of graphical representation and the model is stable under the effect of the three different forces. From the fig. 10 we see that the gravitational force is attractive but both the anisotropic and hydrostatic forces are repulsive and the combine effect of these two forces is counterbalanced by the gravitational force which makes the system in static equilibrium. One interesting thing we can also notice that $F_{h}$ and $F_{a}$ intersects at some point inside the fluid sphere. The causality conditions and the the Herrera's cracking conditions are also satisfied as well. Our model is potentially stable as well since $V_{t}^{2}-V_{r}^{2}<0$ everywhere inside the fluid sphere.

- We have obtained a reasonable bound for the dimensionless quantity $p_{0}$ from the physical analysis and we have shown that it depends on 'a'.

- We have successfully demonstrated the possibility that the anisotropic stars could be ultra compact objects due to the additional support from anisotropy and could be sources of gravitational wave echoes. In this model, we have calculated tidal deformability of the anisotropic stars and show how the tidal love number varies with the compactness factor of a star. As an interesting consequence, we can see that in the black hole limit the love number vanishes. Also, the existence of the non zero value of tidal love number at zero compactness factor agrees with some of the recent findings. 
- Generating function: Lake [52] proposed an algorithm based on the choice of a single monotone function subject to boundary conditions which generates all regular static spherically symmetric perfect-fluid solutions of Einstein equations. Herrera et al. [35] extended this work to the case of locally anisotropic fluids and proved that two functions instead of one is required to generate all possible solutions for anisotropic fluid. To find these two generating function we first consider pressure anisotropy for our present model given as,

$$
\begin{aligned}
p_{t}-p_{r}= & \frac{e^{-\lambda}}{\kappa}\left[\frac{-\lambda^{\prime} \nu^{\prime}}{4}+\frac{\nu^{\prime 2}}{4}+\frac{\nu^{\prime \prime}}{2}-\frac{\lambda^{\prime}-\nu^{\prime}}{2 r}\right. \\
& \left.-\frac{\nu^{\prime}}{r}+\frac{e^{\lambda}-1}{r^{2}}\right] .
\end{aligned}
$$

Now by introducing DB transformation

$$
x=r^{2}, \quad V(x)=e^{-\lambda}, \quad \text { and } \quad y(x)=e^{\nu},
$$

the Eqn. (94), transform to,

$\frac{d V}{d x}\left(1+x \frac{\dot{y}}{y}\right)+V\left[\left(2 \frac{\ddot{y}}{y}-\frac{\dot{y}^{2}}{y^{2}}\right) x-\frac{1}{x}\right]=\kappa \Delta-\frac{1}{x},(95)$

The above equation can be denoted as,

$$
\frac{d V}{d x}+F(x) V=S(x)
$$

which is linear equation of $x$. The integrating factor of the above equation is,

$$
e^{\int F(x) d x}
$$

and the solution of the above equation is,

$$
V(x)=e^{-\int F(x) d x} \int S(x) e^{\int F(x) d x} d x+k,
$$

where $k$ is the constant of integration and

$$
\begin{aligned}
& F(x)=\frac{\left(2 \frac{\ddot{y}}{y}-\frac{\dot{y}^{2}}{y^{2}}\right) x-\frac{1}{x}}{1+x \frac{\dot{y}}{y}}, \\
& S(x)=\frac{\kappa \Delta-\frac{1}{x}}{1+x \frac{\dot{y}}{y}} .
\end{aligned}
$$

From the above discussion it is clear that the model of the compact star stands on two functions $F(x)$ and $S(x)$, where as $F(x)$ and $S(x)$ depends on $y(x)$ and $\Delta$. Therefore for our model the two generating functions are therefore,

$$
\begin{aligned}
y(x)= & \exp \left[\frac { 1 } { 4 } \left\{2 a x+b x^{2}+\frac{2 p_{0}\left(a^{2}+2 b\right)}{b \sqrt{a^{2}-4 b}} \times\right.\right. \\
& \left.\tan ^{-1}\left(\frac{a+2 b x}{\sqrt{a^{2}-4 b}}\right)-\frac{a p_{0}}{b} \log \left(1+a x+b x^{2}\right)\right\} \\
& +B], \\
\Delta= & \frac{x}{4 \Psi^{3}}\left[A_{1}+A_{2} x+A_{3} x^{2}+A_{4} x^{3}+A_{5} x^{4}\right. \\
& \left.+4 a b^{3} x^{5}+b^{4} x^{6}\right] .
\end{aligned}
$$

[1] S Bayin. Anisotropic fluid spheres in general relativity. Phys. Rev. D, 26:1262-1274, Sep 1982.

[2] Piyali Bhar and Farook Rahaman. Search of wormholes in different dimensional non-commutative inspired space-times with Lorentzian distribution. The European Physical Journal C, 74(12):3213, December 2014.

[3] Piyali Bhar, Farook Rahaman, Ritabrata Biswas, and Hafiza Ismat Fatima. Exact solution of a (2 +1 )-dimensional anisotropic star in finch and skea spacetime. Communications in Theoretical Physics, 62(2):221-226, aug 2014.

[4] Piyali Bhar, Ksh. Newton Singh, and Francisco TelloOrtiz. Compact star in Tolman-Kuchowicz spacetime in the background of Einstein-Gauss-Bonnet gravity. The European Physical Journal C, 79(11):922, November 2019.

[5] James Binney and Scott Tremaine. Galactic dynamics. Princeton, NJ, Princeton University Press, 1987, 747 p., 1987.

[6] Bhaskar Biswas and Sukanta Bose. Tidal deformability of an anisotropic compact star: Implications of
GW170817. Phys. Rev. D, 99(10):1-11, 2019.

[7] Suparna Biswas, Dibyendu Shee, B. K. Guha, and Saibal Ray. Anisotropic strange star with Tolman-Kuchowicz metric under $\mathrm{f}(\mathrm{R}, \mathrm{T})$ gravity. The European Physical Journal C, 80(2):175, February 2020.

[8] Richard L. Bowers and E. P. T. Liang. Anisotropic Spheres in General Relativity. Astrophys. J., 188(1):657, 1974.

[9] V. Canuto. Equation of state at ultrahigh densities. Annual Review of Astronomy and Astrophysics, 12(1):167214, 1974.

[10] R. CHAN, M. F. A. DA SILVA, and JAIME F. VILLAS DA ROCHA. Gravitational collapse of self-similar and shear-free fluid with heat flow. International Journal of Modern Physics D, 12(03):347-368, 2003.

[11] R. Chan, L. Herrera, and N. O. Santos. Dynamical instability for shearing viscous collapse. Monthly Notices of the Royal Astronomical Society, 267(3):637-646, 04 1994.

[12] P. K. Chattopadhyay and B. C. Paul. Relativistic star solutions in higher-dimensional pseudospheroidal space- 
time. Pramana - J. Phys., 74(4):513-523, 2010.

[13] Pradip Kumar Chattopadhyay, Rumi Deb, and Bikash Chandra Paul. Relativistic solution for a class of static compact charged star in pseudo-spheroidal spacetime. Int. J. Mod. Phys. D, 21(8):1-24, 2012.

[14] Debabrata Deb, B. K. Guha, Farook Rahaman, and Saibal Ray. Anisotropic strange stars under simplest minimal matter-geometry coupling in the $f(r, \mathcal{T})$ gravity. Phys. Rev. D, 97:084026, Apr 2018.

[15] M.S.R. Delgaty and Kayll Lake. Physical acceptability of isolated, static, spherically symmetric, perfect fluid solutions of Einstein's equations. Comput. Phys. Commun., 115:395-415, 1998.

[16] Saba Fatema and Mohammad Hassan Murad. An Exact Family of Einstein-Maxwell Wyman-Adler Solution in General Relativity. International Journal of Theoretical Physics, 52(7):2508-2529, July 2013.

[17] Tooba Feroze and Azad A. Siddiqui. Charged anisotropic matter with quadratic equation of state. Gen. Rel. Grav., 43:1025-1035, 2011.

[18] M R Finch and J E F Skea. A realistic stellar model based on an ansatz of duorah and ray. Classical and Quantum Gravity, 6(4):467-476, apr 1989.

[19] P. S. Florides. A new interior schwarzschild solution. Proceedings of the Royal Society of London. Series A, Mathematical and Physical Sciences, 337(1611):529535, 1974.

[20] N.K. Glendenning. Compact stars: Nuclear physics, particle physics, and general relativity. ., 1997.

[21] M. K. Gokhroo and A. L. Mehra. Anisotropic spheres with variable energy density in general relativity. General Relativity and Gravitation, 26(1):75-84, March 1994.

[22] Megandhren Govender and Suntharalingam Thirukkanesh. Anisotropic static spheres with linear equation of state in isotropic coordinates. Astrophysics and Space Science, 358, 082015.

[23] Kesh S. Govinder, Megan Govender, and Roy Maartens. On radiating stellar collapse with shear. Mon. Not. R. Astron. Soc., 299(3):809-810, 1998.

[24] Y. K. Gupta and M. K. Jasim. On most general exact solution for Vaidya-Tikekar isentropic superdense star. Astrophys. Space Sci., 272(4):403-415, 2000.

[25] Norman Gürlebeck. No-hair theorem for black holes in astrophysical environments. Phys. Rev. Lett., 114(15), 2015.

[26] Tolga Güver, Patricia Wroblewski, Larry Camarota, and Feryal Özel. The mass and radius of the neutron star in 4u 1820-30. The Astrophysical Journal, 719(2):1807-1812, Aug 2010.

[27] W. Hillebrandt H. Heintzmann. Neutron stars with an anisotropic equation of stare : mass,redshift and stability. Astron. Astrophys., 38:51, 1975.

[28] T Harko and M K Mak. Anisotropy in bianchi-type brane cosmologies. Classical and Quantum Gravity, 21(6):1489-1503, Feb 2004.

[29] T. Harko and M.K. Mak. Anisotropic relativistic stellar models. Annalen der Physik, 11(1):3, Jan 2002.

[30] B. K. Harrison, K. S. Thorne, M. Wakano, and J. A. Wheeler. Gravitation Theory and Gravitational Collapse. Gravitation Theory and Gravitational Collapse, Chicago: University of Chicago Press, 1965, 1965.

[31] L. HERRERA. Physical causes of energy density inho- mogenization and stability of energy density homogeneity in relativistic self-gravitating fluids. International Journal of Modern Physics D, 20(09):1689-1703, Aug 2011.

[32] L Herrera, A Di Prisco, J.L Hernández-Pastora, and N.O Santos. On the role of density inhomogeneity and local anisotropy in the fate of spherical collapse. Physics Letters A, 237(3):113 - 118, 1998.

[33] L. Herrera, A. Di Prisco, J. Martin, J. Ospino, N. O. Santos, and O. Troconis. Spherically symmetric dissipative anisotropic fluids: A general study. Phys. Rev. D, 69:084026, Apr 2004.

[34] L. Herrera, J. Martin, and J. Ospino. Anisotropic geodesic fluid spheres in general relativity. J. Math. Phys., 43:4889-4897, 2002.

[35] L. Herrera, J. Ospino, and A. Di Prisco. All static spherically symmetric anisotropic solutions of einstein's equations. Phys. Rev. D, 77:027502, Jan 2008.

[36] L. Herrera, A. Di Prisco, J. Ospino, and E. Fuenmayor. Conformally flat anisotropic spheres in general relativity. Journal of Mathematical Physics, 42(5):2129, 2001.

[37] L. Herrera, A. Di Prisco, J. Ospino, and E. Fuenmayor. Conformally flat anisotropic spheres in general relativity. Journal of Mathematical Physics, 42(5):2129, 2001.

[38] L. Herrera and N.O. Santos. Local anisotropy in selfgravitating systems. Phys. Rept., 286:53-130, 1997.

[39] A. Hewish, S.J. Bell, J.D.H Pilkington, P.F. Scott, and R.A. Collins. Observation of a rapidly pulsating radio source. Nature, 217:709-713, 1968.

[40] Tanja Hinderer. Tidal Love Numbers of Neutron Stars. Astrophys. J., 677(2):1216-1220, 2008.

[41] A.A. Isayev. General relativistic polytropes in anisotropic stars. Physical Review D, 96(8), Oct 2017.

[42] W. Israel. Singular hypersurfaces and thin shells in general relativity. Il Nuovo Cimento B (1965-1970), 44(1):1-14, July 1966.

[43] W. Israel. Singular hypersurfaces and thin shells in general relativity. Il Nuovo Cimento B (1965-1970), 48(2):463-463, April 1967.

[44] B. V. Ivanov. Maximum bounds on the surface redshift of anisotropic stars. Phys. Rev. D, 65:104011, Apr 2002.

[45] B. V. Ivanov. The importance of anisotropy for relativistic fluids with spherical symmetry. International Journal of Theoretical Physics, 49(6):1236-1243, Mar 2010.

[46] A.J. John and S.D. Maharaj. An Exact isotropic solution. Nuovo Cim. B, 121:27-33, 2006.

[47] Kanti Jotania and Ramesh Tikekar. Paraboloidal spacetimes and relativistic models of strange stars. Int. J. Mod. Phys. D, 15(8):1175-1182, 2006.

[48] Rudolf Kippenhahn and Alfred Weigert. Stellar Structure and Evolution. Astronomy and Astrophysics Library. Springer-Verlag, Berlin Heidelberg, 1990.

[49] K. Komathiraj and S. D. Maharaj. Tikekar superdense stars in electric fields. Journal of Mathematical Physics, 48(4):042501, Apr 2007.

[50] Jitendra Kumar and Y. K. Gupta. A class of new solutions of generalized charged analogues of Buchdahl's type super-dense star. Astrophysics and Space Science, 345(2):331-337, June 2013.

[51] N. O. Santos L. Herrera. JEANS MASS FOR ANISOTROPIC MATTER. Astrophys. J., 438:308, 1995.

[52] Kayll Lake. All static spherically symmetric perfect- 
fluid solutions of einstein's equations. Phys. Rev. D, 67:104015, May 2003.

[53] Kayll Lake. Generating static spherically symmetric anisotropic solutions of einstein's equations from isotropic newtonian solutions. Phys. Rev. D, 80:064039, Sep 2009.

[54] Georges Lemaître. L'Univers en expansion. Annales de la Société Scientifique de Bruxelles, 53, 1933.

[55] Patricio S. Letelier. Anisotropic fluids with two-perfectfluid components. Phys. Rev. D, 22:807-813, Aug 1980.

[56] P. Mafa Takisa, S. D. Maharaj, and Subharthi Ray. Stellar objects in the quadratic regime. Astrophysics and Space Science, 354(2):463-470, Sep 2014.

[57] P. Mafa Takisa, S. Ray, and S. D. Maharaj. Charged compact objects in the linear regime. Astrophysics and Space Science, 350(2):733-740, Jan 2014.

[58] S. D. Maharaj and P. G. L. Leach. Exact solutions for the Tikekar superdense star. Journal of Mathematical Physics, 37(1):430-437, January 1996.

[59] S. D. Maharaj and P. G.L. Leach. Exact solutions for the Tikekar superdense star. J. Math. Phys., 37(1):430437, 1996.

[60] S. D. Maharaj and P. Mafa Takisa. Regular models with quadratic equation of state. General Relativity and Gravitation, 44(6):1419-1432, June 2012.

[61] S. D. Maharaj and S. Thirukkanesh. Generating potentials via difference equations. Mathematical Methods in the Applied Sciences, 29(16):1943-1952, 2006.

[62] S.D. Maharaj and P.Mafa Takisa. Regular models with quadratic equation of state. Gen. Rel. Grav., 44:14191432, 2012.

[63] S.D. Maharaj and S. Thirukkanesh. Generalised isothermal models with strange equation of state. Pramana, 72:481, 2009.

[64] M. K. MAK, PETER N. DOBSON, and T. HARKO. Exact models for anisotropic relativistic stars. International Journal of Modern Physics D, 11(02):207-221, 2002.

[65] M. K. Mak and T. Harko. An Exact Anisotropic Quark Star Model. Chinese J. Astron. Astrophys., 2(3):248259, 2002.

[66] M. K. MAK and T. HARKO. Quark stars admitting a one-parameter group of conformal motions. International Journal of Modern Physics D, 13(01):149-156, Jan 2004.

[67] M. K. MAK and T. HARKO. Quark stars admitting a one-parameter group of conformal motions. International Journal of Modern Physics D, 13(01):149-156, Jan 2004.

[68] M.K. Mak and T. Harko. Anisotropic stars in general relativity. Proceedings of the Royal Society of London. Series A: Mathematical, Physical and Engineering Sciences, 459(2030):393-408, Feb 2003.

[69] S. K. Maurya and Y. K. Gupta. A new class of relativistic charged anisotropic super dense star models. Astrophysics and Space Science, 353(2):657-665, October 2014.

[70] S. K. Maurya, Y. K. Gupta, and Saibal Ray. All spherically symmetric charged anisotropic solutions for compact stars. The European Physical Journal C, 77(6), May 2017.

[71] S. K. Maurya, Y. K. Gupta, Saibal Ray, and Baiju Dayanandan. Anisotropic models for compact stars. The European Physical Journal C, 75(5):225, May 2015.
[72] S Mukherjee, B C Paul, and N Dadhich. General solution for a relativistic star. Classical and Quantum Gravity, 14(12):3475-3480, dec 1997.

[73] S. Mukherjee, B.C. Paul, and N. Dadhich. General solution for a relativistic star. Class. Quant. Grav., 14:34753480, 1997.

[74] Mohammad Hassan Murad. A new well behaved class of charge analogue of Adler's relativistic exact solution. Astrophysics and Space Science, 343(1):187-194, January 2013.

[75] Mohammad Hassan Murad and Saba Fatema. A family of well behaved charge analogues of Durgapal's perfect fluid exact solution in general relativity. Astrophysics and Space Science, 343(2):587-597, February 2013.

[76] Mohammad Hassan Murad and Saba Fatema. A family of well behaved charge analogues of Durgapal's perfect fluid exact solution in general relativity II. Astrophysics and Space Science, 344(1):69-78, March 2013.

[77] Mohammad Hassan Murad and Saba Fatema. Some Exact Relativistic Models of Electrically Charged Selfbound Stars. International Journal of Theoretical Physics, 52(12):4342-4359, December 2013.

[78] Mohammad Hassan Murad and Saba Fatema. Some new Wyman-Leibovitz-Adler type static relativistic charged anisotropic fluid spheres compatible to self-bound stellar modeling. Eur. Phys. J. C, 75(11):1-21, 2015.

[79] N. F. NAIDU, M. GOVENDER, and K. S. GOVINDER. Thermal evolution of a radiating anisotropic star with shear. International Journal of Modern Physics D, 15(07):1053-1065, Jul 2006.

[80] A. Nasim and M. Azam. Anisotropic charged physical models with generalized polytropic equation of state. Eur. Phys. J. C, 78(1):1-9, 2018.

[81] S. A. Ngubelanga and S. D. Maharaj. New classes of polytropic models. Astrophys. Space Sci., 362(3):1-9, 2017.

[82] J. R. Oppenheimer and G. M. Volkoff. On massive neutron cores. Phys. Rev., 55:374-381, Feb 1939.

[83] D. M. Pandya, V. O. Thomas, and R. Sharma. Modified Finch and Skea stellar model compatible with observational data. Astrophys. Space Sci., 356(2):285-292, 2015.

[84] D. M. Pandya, V. O. Thomas, and R. Sharma. Modified Finch and Skea stellar model compatible with observational data. Astrophysics and Space Science, 356(2):285-292, April 2015.

[85] Lk Patel and Sharda S Koppar. A Charged Analogue of the Vaidya?Tikekar Solution. Australian Journal of Physics, 40(3):441, 1987.

[86] A. Rahmansyah, A. Sulaksono, A. B. Wahidin, and A. M. Setiawan. Anisotropic neutron stars with hyperons: implication of the recent nuclear matter data and observations of neutron stars. Eur. Phys. J. C, 80(8), 2020.

[87] S. S. Rajah and S. D. Maharaj. A Riccati equation in radiative stellar collapse. Journal of Mathematical Physics, 49(1):012501, January 2008.

[88] Guilherme Raposo, Paolo Pani, Miguel Bezares, Carlos Palenzuela, and Vitor Cardoso. Anisotropic stars as ultracompact objects in general relativity. Phys. Rev. D, 99:104072, May 2019.

[89] Meredith L. Rawls, Jerome A. Orosz, Jeffrey E. McClintock, Manuel A. P. Torres, Charles D. Bailyn, and Michelle M. Buxton. Refined neutron star mass deter- 
minations for six eclipsing x-ray pulsar binaries. The Astrophysical Journal, 730(1):25, Feb 2011.

[90] Tullio Regge and John A. Wheeler. Stability of a schwarzschild singularity. Phys. Rev., 108(4):1063-1069, 1957.

[91] Clifford E. Rhoades and Remo Ruffini. Maximum mass of a neutron star. Phys. Rev. Lett., 32:324-327, Feb 1974.

[92] Zacharias Roupas and Gamal G.L. Nashed. Anisotropic neutron stars modelling: constraints in Krori-Barua spacetime. Eur. Phys. J. C, 80(10):1-14, 2020.

[93] M. Ruderman. Pulsars: Structure and dynamics. Annual Review of Astronomy and Astrophysics, 10(1):427476, 1972 .

[94] R. F. Sawyer. Condensed $\pi^{-}$phase in neutron-star matter. Phys. Rev. Lett., 29:382-385, Aug 1972.

[95] Karl Schwarzschild. On the gravitational field of a sphere of incompressible fluid according to Einstein's theory. Sitzungsber. Preuss. Akad. Wiss. Berlin (Math. Phys. ), 1916:424-434, 1916.

[96] S.L. Shapiro and S.A. Teukolsky. Black holes, white dwarfs, and neutron stars: The physics of compact objects. ., 1983.

[97] R. SHARMA, S. KARMAKAR, and S. MUKHERJEE. Maximum mass of a class of cold compact stars. International Journal of Modern Physics D, 15(03):405-418, 2006.

[98] R. Sharma and S.D. Maharaj. A Class of relativistic stars with a linear equation of state. Mon. Not. Roy. Astron. Soc., 375:1265-1268, 2007.

[99] R. SHARMA and S. MUKHERJEE. Compact stars: A core-envelope model. Modern Physics Letters A, 17(38):2535-2544, 2002.

[100] R. Sharma, S. Mukherjee, and S. D. Maharaj. General solution for a class of static charged spheres. Gen. Relativ. Gravit., 33(6):999-1009, 2001.

[101] R. SHARMA and B. S. RATANPAL. Relativistic stellar model admitting a quadratic equation of state. International Journal of Modern Physics D, 22(13):1350074, Oct 2013.

[102] R. SHARMA and B. S. RATANPAL. Relativistic stellar model admitting a quadratic equation of state. International Journal of Modern Physics D, 22(13):1350074, 2013.

[103] A. Sokolov. Phase transitions in a superfluid neutron liquid. Sov. J. Exp. Theor. Phys., 52(March 1980):575, 1980.

[104] Jefta Sunzu, Sunil Maharaj, and Subharthi Ray. Charged anisotropic models for quark stars. Astrophysics and Space Science, 352:719, 042014.

[105] Jefta M. Sunzu, Sunil D. Maharaj, and Subharthi Ray. Quark star model with charged anisotropic matter. Astrophysics and Space Science, 354(2):517-524, December 2014.

[106] Jefta M. Sunzu and Mashiku Thomas. New stellar models generated using a quadratic equation of state. Pramana - J. Phys., 91(6):1-10, 2018.

[107] P. Mafa Takisa and S. D. Maharaj. Some charged polytropic models. General Relativity and Gravitation, 45(10):1951-1969, Jul 2013.

[108] S. Thirukkanesh, M. Govender, and D. B. Lortan. Spherically symmetric static matter configurations with vanishing radial pressure. International Journal of Modern Physics D, 24(01):1550002, 2015.
[109] S Thirukkanesh and S D Maharaj. Exact models for isotropic matter. Classical and Quantum Gravity, 23(7):2697-2709, Mar 2006.

[110] S Thirukkanesh and S D Maharaj. Charged anisotropic matter with a linear equation of state. Classical and Quantum Gravity, 25(23):235001, Nov 2008.

[111] S. Thirukkanesh and S. D. Maharaj. Charged relativistic spheres with generalized potentials. Mathematical Methods in the Applied Sciences, 32(6):684-701, Apr 2009.

[112] S Thirukkanesh and F C Ragel. Exact anisotropic sphere with polytropic equation of state. Pramana, 78(5):687-696, 2012.

[113] S. Thirukkanesh and F. C. Ragel. Anisotropic compact sphere with Van der Waals equation of state. Astrophys. Space Sci., 354(2):415-420, 2014.

[114] S. Thirukkanesh, F. C. Ragel, Ranjan Sharma, and Shyam Das. Anisotropic generalization of well-known solutions describing relativistic self-gravitating fluid systems: an algorithm. The European Physical Journal C, 78(1):31, January 2018.

[115] S. Thirukkanesh and F.C. Ragel. Strange star model with Tolmann IV type potential. Astrophys. Space Sci., 352(2):743-749, 2014

[116] V. O. THOMAS and B. S. RATANPAL. Non-adiabatic gravitational collapse with anisotropic core. International Journal of Modern Physics D, 16(09):1479-1495, 2007.

[117] V. O. THOMAS, B. S. RATANPAL, and P. C. VINODKUMAR. Core-envelope models of superdense star with anisotropic envelope. International Journal of Modern Physics D, 14(01):85-96, 2005.

[118] Kip S. Thorne. Tidal stabilization of rigidly rotating, fully relativistic neutron stars. Phys. Rev. D - Part. Fields, Gravit. Cosmol., 58(12):1-9, 1998.

[119] Ramesh Tikekar. A class of static charged dust spheres in general relativity. General Relativity and Gravitation, 16(5):445-451, May 1984.

[120] Ramesh Tikekar. Exact model for a relativistic star. J. Math. Phys., 31(10):2454-2458, 1990.

[121] Ramesh Tikekar. Exact model for a relativistic star. Journal of Mathematical Physics, 31(10):2454-2458, October 1990.

[122] Ramesh Tikekar and V. O. Thomas. Relativistic fluid sphere on pseudo-spheroidal space-time. Pramana - J. Phys., 50(2):95-103, 1998.

[123] Ramesh Tikekar and V. O. Thomas. Relativistic fluid sphere on pseudo-spheroidal space-time. Pramana - J. Phys., 50(2):95-103, 1998.

[124] Ramesh Tikekar and V. O. Thomas. Anisotropic fluid distributions on pseudo-spheroidal spacetimes. Pramana - J. Phys., 52(3):237-244, 1999.

[125] Ramesh Tikekar and V. O. Thomas. A relativistic coreenvelope model on pseudospheroidal space-time. Pramana - J. Phys., 64(1):5-15, 2005.

[126] Richard C. Tolman. Static solutions of einstein's field equations for spheres of fluid. Phys. Rev., 55:364-373, Feb 1939.

[127] Vladimir V. Usov. Electric fields at the quark surface of strange stars in the color-flavor locked phase. Phys. Rev. D, 70:067301, Sep 2004.

[128] P. C. Vaidya and Ramesh Tikekar. Exact relativistic model for a superdense star. J. Astrophys. Astron., 3(3):325-334, 1982. 
[129] F Weber. Pulsars as Astrophysical Laboratories for Nuclear and Particle Physics. Institute of Physics Publishing, Bristol, 1999.

[130] F. Weber, R. Negreiros, P. Rosenfield, and M. Stejner. Pulsars as astrophysical laboratories for nuclear and particle physics. Progress in Particle and Nuclear Physics, 59(1):94-113, Jul 2007.

[131] Ya. B. Zeldovich and Igor D. Novikov. Relativistic as- trophysics. Vol.1: Stars and relativity. ., 1971.

\section{ACKNOWLEDGEMENT}

PB is thankful to IUCAA, Pune, Govt of India for providing visiting associateship. SD gratefully acknowledges support from the Inter-university Centre for Astronomy and Astrophysics (IUCAA), Pune, India, where a part of this work was carried out under its Visiting Research Associateship Programme. 


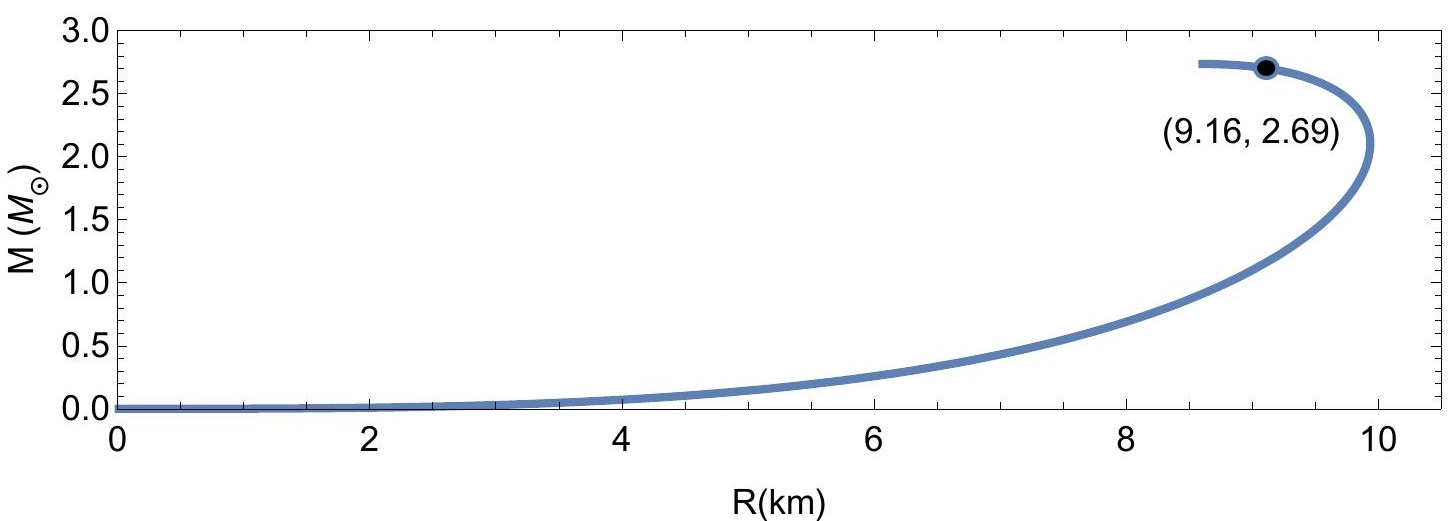

